\title{
Neural Mechanisms of Motion Detection, Integration, and Segregation: From Biology to Artificial Image Processing Systems
}

\author{
Jan D. Bouecke, ${ }^{1}$ Emilien Tlapale, ${ }^{2}$ Pierre Kornprobst, ${ }^{2}$ and Heiko Neumann ${ }^{1}$ \\ ${ }^{1}$ Faculty of Engineering and Computer Sciences, Institute for Neural Information Processing, Ulm University, James-Franck-Ring, \\ 89069 Ulm, Germany \\ ${ }^{2}$ Equipe Projet NeuroMathComp, Institut National de Recherche en Informatique et en Automatique (INRIA), \\ Unité de recherche INRIA Sophia Antipolis, Sophia Antipolis Cedex, 06902, France \\ Correspondence should be addressed to Heiko Neumann, heiko.neumann@uni-ulm.de
}

Received 15 June 2010; Accepted 2 November 2010

Academic Editor: Elias Aboutanios

Copyright ( 2011 Jan D. Bouecke et al. This is an open access article distributed under the Creative Commons Attribution License, which permits unrestricted use, distribution, and reproduction in any medium, provided the original work is properly cited.

Object motion can be measured locally by neurons at different stages of the visual hierarchy. Depending on the size of their receptive field apertures they measure either localized or more global configurationally spatiotemporal information. In the visual cortex information processing is based on the mutual interaction of neuronal activities at different levels of representation and scales. Here, we utilize such principles and propose a framework for modelling neural computational mechanisms of motion in primates using biologically inspired principles. In particular, we investigate motion detection and integration in cortical areas V1 and MT utilizing feedforward and modulating feedback processing and the automatic gain control through center-surround interaction and activity normalization. We demonstrate that the model framework is capable of reproducing challenging data from experimental investigations in psychophysics and physiology. Furthermore, the model is also demonstrated to successfully deal with realistic image sequences from benchmark databases and technical applications.

\section{Introduction and Motivation}

A key visual competency of many species, including humans, is the ability to rapidly and accurately ascertain the sizes, locations, trajectories, and identities of objects in the environment. For example, noticing a deer moving behind a thicket, or steering around obstacles through a crowded environment, indicates that many of the tasks of vision serve as a basis to guide behaviour based on the spatiotemporally changing visual input. The analysis and interpretation of moving objects based on motion estimations is thus a major task in everyday vision. However, motion can locally be measured only orthogonal to an extended contrast (aperture problem), while this ambiguity can be resolved at localized image features, such as corners or junctions from nonoccluding geometrical configurations. Several models have been suggested that focus on the problem of how to integrate localized and mostly ambiguous local motion estimates. For example, the vector sum approach averages movement vectors measured for a coherent shape [1]. Local motion signals of an object define a subspace of possible motion interpretations, namely, the so-called motion constraint equation (MCE; [2]). If several distinct measures are combined, their associated constraint lines in the velocity space intersect and thus yield the velocity common to the individual measures (intersection of constraints, IOC) $[3,4]$. Bayesian models combine different probabilities for velocities and combine these estimates with statistical priors which often prefer slower motions $[5,6]$ (Simoncelli [7]). Like for the IOC, Bayesian models mostly assume that motion estimates belonging to distinct objects were already grouped together. Unambiguous motion signals can be measured at locations of significant 2D image structure such as curvature maxima, corners, or junctions. These sparse features can be tracked over several frames to yield robust movement estimates and predictions (feature tracking) [8]. Coherent motion is often computed by utilizing an optimization approach in which the solution is searched given a set of measurements that minimizes the distance to the constraint lines in a least squares sense [4]. Other approaches utilize 

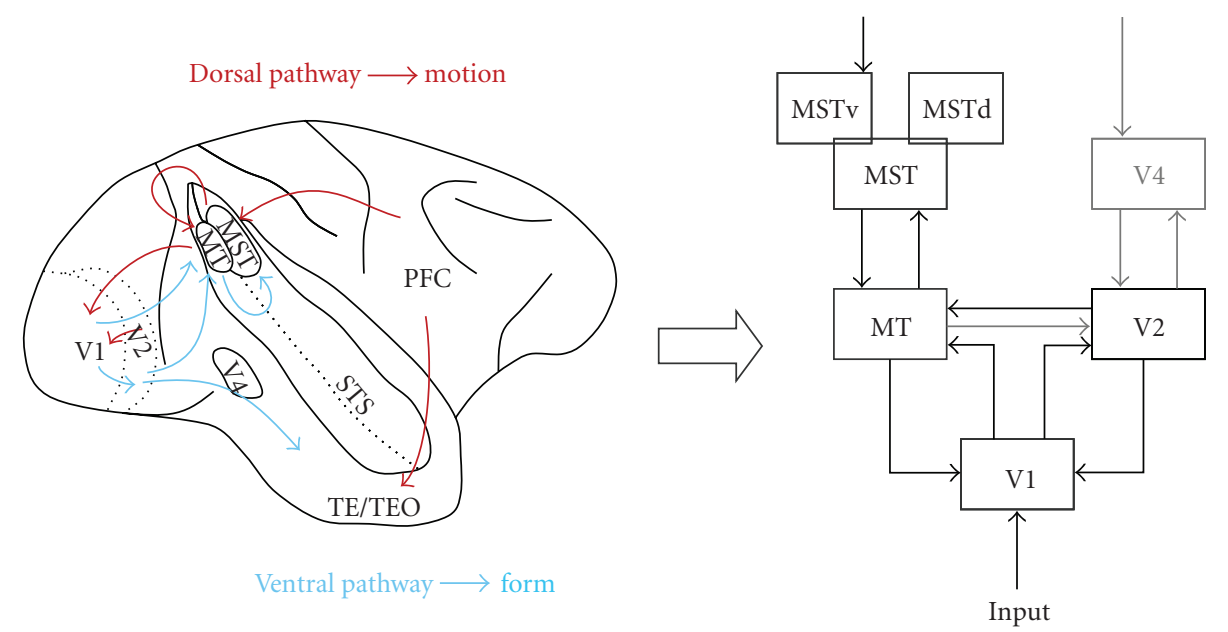

FIGURE 1: Structure of the organization of the visual cortical architecture with its areas and interconnections. The entry stage for cortical visual processing is in area V1, the primary visual cortex. Feature processing along ascending pathways (blue arrows) proceeds along two roughly segregated pathways, namely, the dorsal and the ventral pathway, respectively. While the processing along the ventral path is mostly devoted to shape and form (WHAT system), the dorsal path is mostly concerned with motion processing (WHERE system). Areas higher up in the hierarchy send feedback connections along descending pathways (red arrows) to influence the activation distributions at earlier stages in the hierarchy. The scheme of interactive processing between different areas has been sketched on the right in a box-and-arrow scheme. The different arrows indicate the signal flow between the different boxes, namely, areas, in the layout. Several cortical areas are highlighted here to allow an association with major cortical areas and also the cross-reference between the brain sketch on the left and the box picture on the right (V1: primary visual cortex; MT: medial temporal; MST: medial superior temporal (with v and d denoting the ventral and dorsal subdivisions, resp.); PFC: prefrontal cortex; V2: secondary visual area; V4: visual area 4; TE/TEO: areas in inferior temporal cortex; STS: superior temporal sulcus).

a priori models that impose smoothness upon the set of possible solutions of the desired flow field in homogeneous regions [2, 9] or along surface boundaries [10].

Here, we investigate a different route by studying the mechanisms of the primate visual system to process visual motion induced by moving objects or self-motion. Motion information is primarily processed along the dorsal pathway in the visual system, but mutual interactions exist at different stages between the dorsal and ventral pathways [11]. As outlined in Figure 1 the different pathways are instantiated by a hierarchy of interacting areas with different functional competencies which is exemplified by the box-and-arrow conceptualization in the right part of the sketch. In this paper, we will focus on the integration and segregation of visual motion in reciprocally connected areas V1 and MT by proposing a dynamical model to provide a simple framework for $2 \mathrm{D}$ motion integration. We utilize a simple set of computational properties that are common in biological architectures. We consider feedforward and feedback connectivities between layered representation of cells operating at different scales or spatial resolutions. Low-level cues for visual surface properties can be combined with representations at a more global scale that incorporates context information and knowledge by reentering activity from representations higher up in the processing hierarchy to selectively modulate or bias the computations at the lower scales. Despite its simplicity, the model is able to explain experimental data and, without parameter changes, to successfully process realworld data used for model benchmarking [12, 13]. In all, the paper summarizes some previous work of the authors, namely, work of [14-17] by using a common framework of model description. Most importantly, the framework has been extended such that different neural interaction schemes can be utilized in different variants of the model. This development allows relating the modelling framework to recent proposals concerning normalization mechanisms in vision to account for nonlinearities in processing as observed in different cortical areas (e.g., [18]).

The paper is organized as follows. In Section 2 we outline the approach to neural modelling based on the population level of neuronal activity and gradual activation dynamics. Section 3 is built upon the general modelling framework and describes the neural model of motion estimation. Readers who are interested primarily in the motion model but not in the general modelling framework might skip Section 2 and proceed directly to Section 3. In Section 4 we present various simulation results that highlight the neural principles used for motion computation. A discussion of the major contributions and relations to previous work is presented in Section 5. The paper concludes with a brief summary in Section 6.

\section{Neural Modeling Approach}

2.1. Neurodynamics and Notational Formats. The basic processing units in biological information processing are individual neurons. In cortical areas they are organized into different areas each of which shows a typical layering. Cortical areas are organized into six layers which are characterized 
by cell clustering, their lateral interconnectivities, and the major terminations of input and output fiber projections. The transmission of activity in neurons is denoted in terms of potential changes across the membrane of a cell. Single cell dynamics can be described at various levels of detail, for example, at the level of multicompartments, as a single compartment entity or as a cascade model ([19]; see their Figure 1). Here, we utilize single compartment models of neurons, which are essentially point-like representations of a neuron neglecting influences from widespread dendrites and related nonlinear interactions. The membrane acts both as a resistor (that blocks ions of different types to freely pass across the barrier) and as a capacitance to build a charge at both sides of the membrane. Without any input current the cell membrane is in a state of dynamic equilibrium in which currents are flowing across the membrane that balance each other, resulting in zero net current flow. Gates that have constant or activity dependent conductances allow different amounts of ions passing the membrane to change its potential. A simple description of a piece of membrane takes into account the conductance $C$, the resistance $R$, and the resting potential $v$, resembling an $R C$ circuit. By applying Kirchhoff's laws we can specify the dynamics of the membrane potential (voltage) given arbitrary input currents.

If we take into account excitatory and inhibitory synaptic inputs that are delivered by fast chemical synapses, then the respective synaptic currents need to be incorporated in the dynamic voltage equation. This leads to the following dynamics:

$$
\tau \frac{d v(t)}{d t}=-v(t)+R \cdot g_{\mathrm{ex}} \cdot\left(E_{\mathrm{ex}}-v(t)\right)+R \cdot g_{\text {in }} \cdot\left(v(t)-E_{\text {in }}\right),
$$

where $\tau=R C$ defines the membrane constant, $g_{\text {ex }}$ and $g_{\text {in }}$ denote time-varying and input dependent membrane conductances (separate for excitatory and inhibitory synapses, resp.), and $E_{\text {ex }}$ and $E_{\text {in }}$ denote saturation points defining the respective reversal battery potentials. If the net effect of synaptic inputs causes a depolarization of the cell exceeding a certain threshold level, then the cell emits a spike. This behaviour has been captured in simplified models of leaky integrate-and-fire (LIF) models [20]. The spatiotemporal signature of spiking response pattern of groups of neurons is believed to provide the neural code for sensory processing. While we believe that the temporal dimension of spiking behaviour is important to achieve robust feature integration of patterns in a distributed fashion (see, e.g., $[21,22]$ ), we focus here on the average behaviour of neurons or groups of neurons. The model neurons investigated here consider the (average) firing rate to encode the strength and significance of input stimuli along their feature dimensions.

Grossberg [23] summarized and unified various proposals to describe the neural response properties by using a generalized notation of the membrane equation, namely,

$$
\begin{aligned}
\tau \frac{d v(t)}{d t}= & -A \cdot v(t)+(B-C \cdot v(t)) \cdot \text { net }_{\mathrm{ex}} \\
& -(D+E \cdot v(t)) \cdot \text { net }_{\mathrm{in}},
\end{aligned}
$$

which is the basis for the notational format used in this contribution. Here the constant $A$ denotes the rate of passive activity decay when the external input is switched off. The introduction of parameters $B$ and $D$ allows transforming parts of this generic equation into additive components by eliminating the shunts, such as in the case of additive centersurround interactions.

Saturation properties can be investigated by the steadystate solution of (2) (for simplicity, we assume here that the net input is generated by feedforward signals). We get

$$
v_{\infty}=\frac{B \cdot \text { net }_{\text {ex }}-D \cdot \text { net }_{\text {in }}}{A+C \cdot \text { net }_{\text {ex }}+E \cdot \text { net }_{\text {in }}} .
$$

The limits for increasing excitatory input by pushing its activity to infinity determine an upper bound $v_{\uparrow}(t)=B / C$, while increasing the inhibitory input approaches a lower bound, $v_{\downarrow}(t)=-D / E$. This property establishes a bounded input/bounded output property for the activation of a model cell (or group of model cells).

We can also assess the activation properties in standard operation conditions when the activation is far from saturation points and the input is in moderate range (for simplicity we assume constant settings for parameters $C$ and $E$, namely, $C=E=1$ ). Closer inspection of (2) shows that the conductance changes for excitatory and inhibitory inputs, respectively, are approximately linear. To put it differently, under the conditions outlined the approximate conditions $B-v(t) \approx c_{\mathrm{ex}}$ and $D+v(t) \approx c_{\text {in }}$ hold. As a consequence, (2) simplifies to the following linear equation:

$$
\tau \frac{d v(t)}{d t}=-A \cdot v(t)+c_{\mathrm{ex}} \cdot \text { net }_{\mathrm{ex}}-c_{\mathrm{in}} \cdot \text { net }_{\mathrm{in}}
$$

under these conditions. Equation (4) demonstrates that the rate of change in response is governed by an approximately linear property and saturates for increased steady input.

\subsection{Cascade Architecture and Description of Generic Cortical} Processing Stages. Our modelling of neural mechanisms (functionality) and their interaction is motivated by principle findings of electrophysiology, anatomical studies, and theories of information processing of macaque monkey's brain. We follow the principle that mechanisms of neural processing are distributed and hierarchically organized in different areas of visual cortex which are partly bidirectional connected. Van Essen and Gallant [11] identified numerous visual and visually associated areas with significant connectivity. A second principle states that each visual area adds a specific type of functionality like the extraction of a (task relevant) feature. We consider several interconnected visual areas that are included in the model. In previous work, on which this research is based, several areas are considered that are relevant to the given visual task. For example, a grouping mechanism that has been proposed to enable the enhancement and extraction of oriented visual structure mainly involves the first two stages along the ventral pathway, namely, cortical areas V1 and V2 [24]. In a similar fashion, texture boundary detection has been investigated involving areas V1, V2, and V4 [25-27] again using the same 


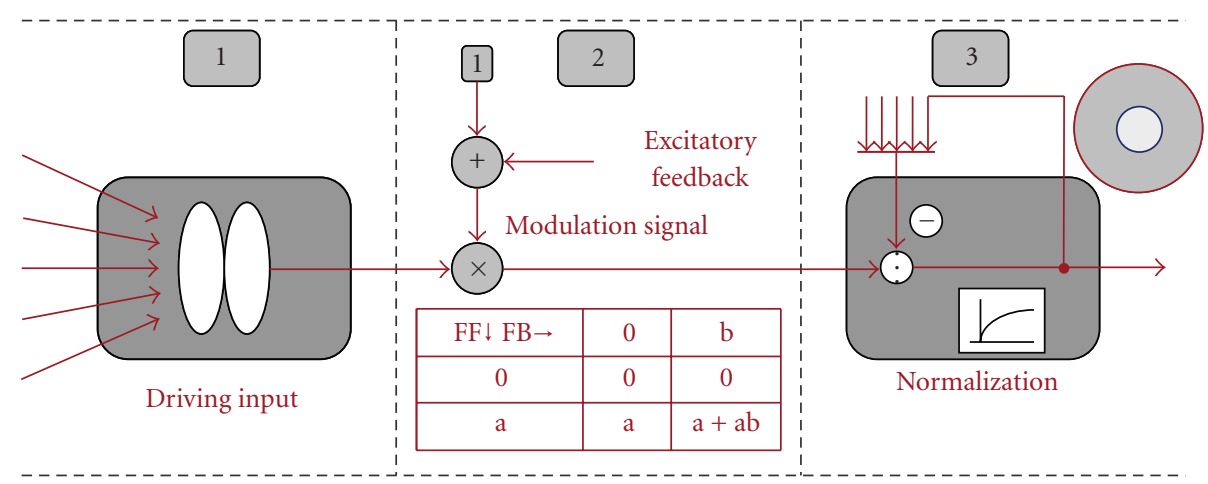

FIgURE 2: Three-stage cascade of dynamical processing stages used to determine the activation level of cells in one model area. Stage 1 (left) pools the bottom-up input signal by a filter mechanism that implements the respective cells' receptive field properties. The resulting activity is fed forward through the next stages of the cascade. Stage 2 (middle) realizes a multiplicative feedback interaction from higher model areas to modulate the initial activation from the filtering stage. This mechanism implements a linking strategy in which the feeding input is required to drive the response, while feedback signals can only modulate the driving input. Feedback cannot by itself generate any new activation. On the other hand, the lack of feedback does not lead to the extinction of activities along the feedforward path such that these activities are left unchanged. In Stage 3 (right) the top-down modulated activity undergoes a stage of shunting on-center/off surround competition over a neighborhood in the spatial and feature domain.

connection and interaction structure. Here, we investigate the analysis of visual motion, again based on the interaction of several areas, but now along the dorsal pathway. The details will be explained in Section 3.

In cortex, anatomically different structures and interconnections can be distinguished in six layers. These layers contribute to realize the computational function of a given area. We employ a simplified, thus more abstract, description of the layered architecture at each cortical stage, or area. In the model, we emphasize key principles of interactive processes that make three different hierarchically organized stages. In particular, we suggest a generic three-level processing cascade that is motivated by layered processing within visual cortex which is sketched in Figure 2.

Before specifying details of the different stages of the model architecture, we like to emphasize the functional logic of the cascade. Assume that the initial stage of processing, or filtering, generates a representation with the driving input activation (stage 1 of Figure 2). Now consider the output of the cascade which generates a normalized representation of activities (stage 3). Such normalization, in a nutshell, keeps the overall energy in the local region mainly constant, so that individual activities balance their activation against the other activities in a region of the visual field that is covered by the neighbourhood in space and feature domain under consideration. Now consider the function of modulatory feedback (stage 2 ). If the activity at a given position in space and feature domain is enhanced by excitatory feedback, then the activity is increased by a component that is proportional to the correlation between feeding input and the modulatory feedback signal amplitude. If no feedback is present, the driving input is left unchanged. Now, reconsider the final stage of normalizing the activity in the pool of cells. Since this mechanism tends to keep the total energy within limits, any prior amplification will, in turn, inhibit those cells and their activation that have not received any input via modulatory feedback signals. Thus, the net effect of modulatory signal enhancement and subsequent competition implements the belief accumulation for a feature response at a target location and the reduction of the likelihood for a representation that does not receive any support (derived from a broader visual context).

The three stages of the cascade will now be sketched and discussed in more detail.

(1) The first processing stage includes a spatial integration and nonlinear enhancement of the signal, which is realized through synaptic signal processing in the dendritic tree laterally integrating incoming feeding signals [28]. In other words, the initial stage of the cascade acts like a filter that can be linear or non-linear in principle. For example, in area V1 orientation selective filters, or simple cells, measure the presence of local oriented contrasts. At other stages, like areas V2 or V4, long-range integration of inputs establishes oriented boundaries, while coarse-grain lateral interaction senses the presence of orientation discontinuities in texture patterns. In motion, such input filtering in V1 measures initial direction-selective spatiotemporal changes or integrates such estimates into directional motion responses in area MT [29].

(2) In the second processing stage, feedback (FB) signals reenter that are delivered by other visual areas, possibly from stages higher up in the hierarchy. Such feedback is modulatory as it cannot by itself generate activation without the presence of feeding, or driving, input. The table in Figure 2 outlines the logic of processing at this stage in the cascade. Each row summarizes the situation of presence or nonpresence of feeding input (zero level or activity a) while the columns denote the situation for feedback signals (zero feedback signal or feedback signal b). The interaction realizes a linking strategy as originally proposed by [30]. In a nutshell, when no driving input is present, then even the presence of feedback activity cannot generate any net response. However, if driving input is present but receives no feedback signal, then the input is not extinguished by simple 
multiplicative combination. Rather, the feeding input is left unchanged. Only in the case when both feeding input as well as modulating feedback signals exist, then the feedforward signal is enhanced by a multiplicative gain control. We suggest a simple mechanism that is denoted by out $\mathrm{x}_{\mathbf{x}, \text { eat }}=$ drive $_{\mathbf{x} \text {,feat }} \cdot\left(1+\lambda \cdot\right.$ feedback $\left._{\mathbf{x} \text {,feat }}\right)$, where $\lambda$ defines a constant amplification factor (indices ( $\mathbf{x}$, feat) denote the spatial position and the feature that is considered, e.g., velocity or contrast orientation). If the feedback signal is generated by mechanisms that cover a large spatial region and combine multiple input streams, then this allows context information to be reentered to earlier stages of processing and the representations created there. Such contextual modulation effects may contribute to texture segmentation (Zipser et al. [31]), figure-ground segregation [32], and motion integration. In all, such feedback is a powerful mechanism for selective tuning of sensory and processing stages in a distributed and hierarchical processing scheme as reflected in the scheme of hierarchical organization of visual areas (Bullier [33]).

(3) With the third processing stage the integrated signals are normalized by lateral interaction between retinotopic organized features. Lateral (horizontal) connections often build the surround of a receptive field's integrating area (Stettler et al. [34]). Following the suggestion of Sperling [35] lateral interaction incorporates a normalization that has the effect to bound activity. This inhibitory lateral interaction is implemented by dividing activity at each retinotopical location by laterally integrated input activity, net ${ }^{\text {in }}$. This property is achieved in the model by the saturation properties of the model membrane conductances as denoted in (2). By setting parameters $C=D=0$ (2) simplifies to

$$
\tau \frac{d v(t)}{d t}=-A \cdot v(t)+B \cdot \text { net }_{\text {ex }}-E \cdot v(t) \cdot \text { net }_{\text {in }}
$$

which equilibrates to

$$
v_{\infty}=\frac{B \cdot \text { net }_{\mathrm{ex}}}{A+E \cdot \text { net }_{\mathrm{in}}} .
$$

We assume that the net inputs are calculated by an oncenter and off-surround mechanism, with net ex $=$ act $*$ $\Lambda^{\text {center }}$ and net $_{\text {in }}=$ act $* \Lambda^{\text {surround }}$, “ $*$ " denoting the convolution operator. Then, the surround input acts on the center input activation by a divisive effect. It should be noted that the effect can be amplified by allowing small subtractive inhibition from surround input level to act on the center activation (setting $D>0$ ). This leads to contrast enhancement which is still normalized by the surround input activation.

The generic flow of input signals that incorporates excitatory and inhibitory driving input specifies the onand off-subfields of a model cell. In addition to this, Carandini and coworkers found evidence for characteristic nonlinearities in the response characteristics of cortical cells, namely, orientation selective V1 cells. These nonlinearities capture miscellaneous effects including (i) contrast responses which show saturation properties at different levels, and (ii) nonspecific suppression by stimuli which do not, by themselves, lead to any cell firings. These include crossorientation inhibition and nonspecific suppression that is (largely) independent of motion, orientation, spatial, and temporal frequency (as well as an increase of contrast leading to faster response). Also, (iii) nonlinearities were observed in which spatial summation of cells changes with stimulus contrast [18]. The authors suggest that a stage of (delayed) divisive inhibition by unspecific pooling of neuron responses over a large neighbourhood in space and feature domain can account for this nonlinearity $[18,36]$. Figure 3 summarizes the components of the model of a cortical cell and its possible biophysical implementation by the mechanism denoted in (2). Here, the excitatory and inhibitory driving inputs regulate the conductances of the model cell's membrane, namely, $g_{\text {ex }}$ and $g_{\text {in }}$, respectively, while the passive (constant) leakage conductance realizes the decay of activation to a resting state in the case of lack of input. The incorporation of an additional shunting conductance, $g_{\text {shunt }}$, that is regulated by the average activation from a pool of neurons in the same cortical layer leads to the divisive normalization of cortical activity (gray shaded component in the extended circuit model of Figure 3). Note that in the original proposal by Carandini and Heeger [36] this component also incorporated a battery, $E_{\text {shunt }}$, that allows an additional additive influence of the pooled activation on the target cell. We omit this here, because the pooling is considered to generate a silent outer-surround effect. The outer-surround is defined by a spatial region around a target cell that is supposed to have an inhibitory effect on the target cell's response. If the inhibition is purely divisive, then it does not generate a measurable effect as long as the target cell is inactive. This divisive, or silent, inhibition effect is driven by the surround region defining the pool of cells to normalize the cell activities governed by the outer surround region.

In all, the extended circuit constitutes the so-called normalization model of cortical cell responses. It is important to clarify the individual contributions of the input activities. The net excitatory and inhibitory input is thought to be generated by the filtering mechanism at the initial stage of the cascade architecture (see above). So, the input activity feeds the excitatory and inhibitory subfields, for example, on-center and off-surround, of a given target cell that shows a saturation of its activity when the input is pushed to the limits. The normalization property is controlled by the pool of cells of a similar type like the target cell. The range of spatial integration for the pooling is supposed to be much larger than the spatial range of the excitatory/inhibitory integration. As a consequence, the normalization by the pooled activation regulates the overall activity of the cells by keeping the total response energy approximately constant. The dynamics is governed by the following mutually coupled pair of equations:

$$
\begin{aligned}
\tau \frac{d v(t)}{d t}= & -E_{\mathrm{decay}} \cdot v(t)+\left(E_{\mathrm{ex}}-v(t)\right) \cdot \text { net }_{\mathrm{ex}} \\
& -\left(E_{\mathrm{in}}+v(t)\right) \cdot \text { net }_{\mathrm{in}}-\alpha \cdot v(t) \cdot w^{\mathrm{pool}}(t),
\end{aligned}
$$




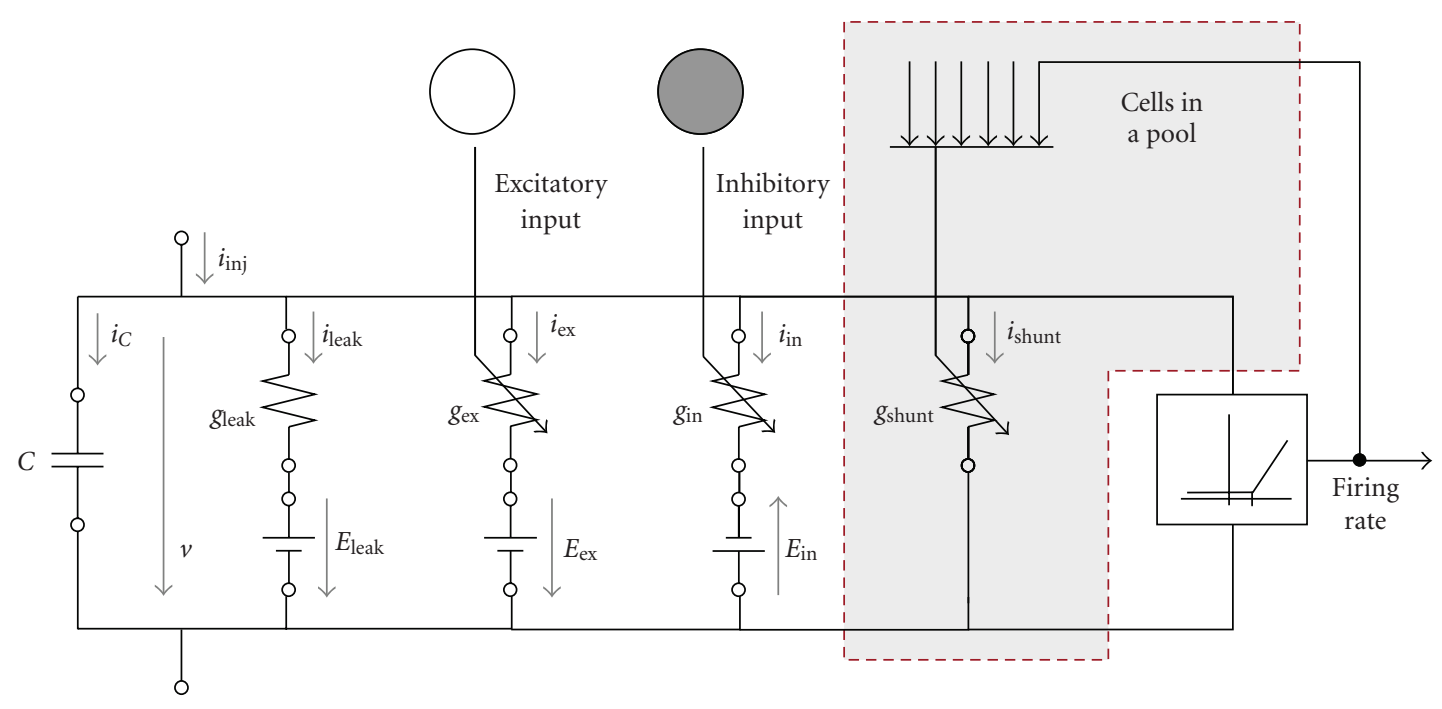

FIGURE 3: Circuit model to describe the dynamics of the membrane potential of a model cell. Simple single compartment models of neurons describe the membrane as a layered patch of phospholipid molecules that separate the internal and external conducting solution acting as an electrical capacitance. The membrane is an electrical device consisting of a capacitance, $C$, a specific membrane resistance, $R$, and a resting potential driven by a battery $\left(E_{\text {leak }}\right)$. The model takes into account excitatory and inhibitory synaptic input currents to adaptively change the membrane conductance denoted by $g_{\mathrm{ex}}$ and $g_{\mathrm{in}}$, respectively. The regulation of the membrane conductance by silent, or shunting, inhibition, $g_{\text {shunt }}$, through the activity from a pool of cells is depicted by the component on the right (grey shaded region). See text for further details and discussion.

$$
\begin{aligned}
\tau_{\mathrm{pool}} \frac{d w^{\mathrm{pool}}(t)}{d t}= & -w^{\mathrm{pool}}(t)+\left(E_{\mathrm{ex}}^{\mathrm{pool}}-w^{\mathrm{pool}}(t)\right) \\
& \times\left\{v(t) * \Lambda^{\mathrm{pool}}\right\}
\end{aligned}
$$

with $\Lambda^{\text {pool }}$ denoting the integration kernel for the pooling of activities and $\alpha$ is a constant amplification. Since the pooled activity enters the shunting inhibition mechanism, the response property becomes nonlinear. The components displayed in Figure 3 relate to the elements in (7) in the following way: conductances $g_{\mathrm{ex}}, g_{\text {in }}$, and $g_{\text {shunt }}$ are denoted here by net ex $_{\text {net }}$, and $w_{\text {pool }}$, respectively ( $w_{\text {pool }}$ is computed separately in the second part of the equation); $g_{\text {leak }}$ is constant denoted by $E_{\text {decay }}$. The resting level for the passive decay is assumed to be zero such that the battery $E_{\text {leak }}=0$. The constant $\tau=R C$ is defined by the membrane capacitance and the resistance $R=1 / g_{\text {leak }}$.

\section{Model of Motion Processing in Cortical Architecture}

3.1. Three-Level Cascade in Motion Analysis. The generic cascade architecture as discussed in the previous section has been specifically established for a model of motion detection and integration along the first stages of the dorsal cortical pathway. The core model architecture consists of essentially two model areas, namely, area V1 and MT. A sketch of our model architecture for motion processing is presented in Figure 5 which consists of two main model areas. Motion analysis in visual cortex starts with primary visual area $\mathrm{V} 1$ and is subsequently followed by parietal areas such as MT/MST and beyond. These areas communicate with a bidirectional flow of information via feedforward and feedback connections. The mechanisms of this feedforward and feedback processing between model areas V1 and MT can be described by a unified architecture of lateral inhibition and modulatory feedback whose elements are outlined in the previous Section 2.2. Here, we present the model dynamics within and between model cortical areas V1 and MT involved to realize the detection and integration of locally ambiguous motion input signals.

In a nutshell, following the general outline in the previous Section 2.2, the model consists of two areas with similar architecture that implement the following mechanisms (compare Figure 4).

(1) Input Filtering Stage. Feedforward motion detection and integration is considered as a (non-) linear filtering stage to process spatiotemporal input patterns to generate the driving, or feeding, input activation for each model area at the initial stage of the 3level-cascade. The activity generates the driving, or feeding, input activities which are denoted by lines with arrow heads in Figure 4.

(2) Modulating Feedback. Cells in model area V1 that represent the initial motion response are modulated by cell activations from model area MT. Cells in MT can, in principle, also be modulated by higher areas such as MST or attention. Since we focus here on the two stages of V1-MT interactions, the feedback signal path entering model area MT is set to zero. In order to distinguish the modulating property that cannot generate an activity without coexisting input, we denote it by a dashed line with arrow head (Figure 4 ). 


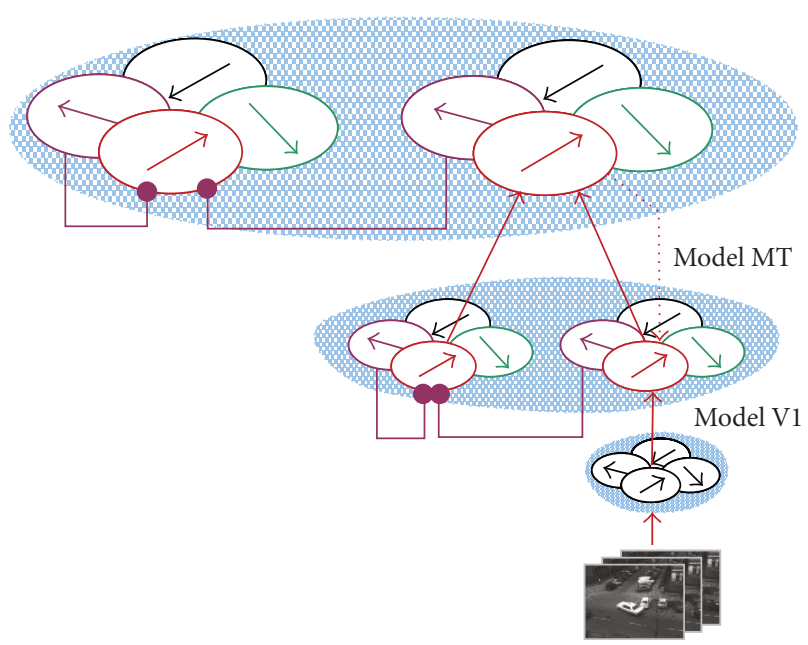

FIGURE 4: Schematic view of the model showing the interactions of the different cortical stages that were taken into account by the model. In essence, it is shown how initial motion is detected and further processed at the stage of area V1. V1 activity is fed forward (red lines with arrow heads) to be integrated by motion selective cells in model area MT. Such cells integrate over a larger spatial neighbourhood and thus build an increasing spatial scale. Cells in $\mathrm{V} 1$ as well as in MT interact via inhibitory connections (purple lines with round heads). Feedback from MT to V1 (red dashed lines with arrow heads) connects cells of corresponding selectivity in the motion feature domain.

(3) Lateral Interaction and Normalization. The final stage of the cascade implements a center-surround architecture with saturation property to normalize the overall activation from the inputs. The process can be augmented by the normalization from the pool of neurons in the same layer of the area under consideration. The laterally inhibitory interactions are denoted by lines with rounded heads (Figure 4).

The model describes the interactions between several layers processing local motion information. The state of each layer is described by a scalar-valued function corresponding to an activation level at each spatial position and for each velocity (speed and direction). The model estimates the velocity information from an input grey level video sequence utilizing the mapping $I:(\mathbf{x}, t) \in \Omega \times \mathbf{R}^{+} \rightarrow I(\mathbf{x}, t) \in \mathbf{R}$, where $\mathbf{x}=(x, y)$ denotes spatial positions in the 2D image domain $\Omega$ and $t$ is the time. The motion responses $y$ in the different stages $i \in\{1,2,3\}$ are denoted by the following equation:

$$
\begin{array}{r}
y_{i}:(\mathbf{x}, \mathbf{v e l}, t) \in \Omega \times \Upsilon \times \mathbf{R}^{+} \longrightarrow y_{i}(\mathbf{x}, \mathbf{v e l}, t) \\
\in[0, B], \\
i=0,1,2
\end{array}
$$

where vel $=(s, \phi)$ denotes the $2 \mathrm{D}$ velocity space composed of speed and direction and $i$ indexes the computational stage within the 3 -level cascade in a model area. The responses $y_{i}$ at different stages are bounded to keep activations levels between 0 and a maximum level denoted by the constant $B$. In Figure 5 the hierarchy of model areas related to the initial stages of cortical motion processing is outlined in a box-andarrow display. In a nutshell, the input signal is processed by some filtering stage, for example, in order to preprocess the input. This stage is associated with Retina and/or LGN. In Figure 5 the filtering stages are displayed by the small icons corresponding to the cell receptive fields and their velocity selectivities.

The following stages define the core elements of the computational model as proposed in this paper. The initial motion-selective filtering in model area $\mathrm{V} 1$ is realized by a spatiotemporal correlation scheme. We employed an extended Reichardt detector (compare [14]) but have also utilized spatiotemporal filtering mechanisms in order to deal with spatial and temporal scales (compare [37]). The initial motion estimation mechanism is detailed in the following. The mechanisms for further processing of detected motion signals and their integration are associated with areas V1 and MT. Figure 5 displays this by indicating the first stage of representations with direction selective units and the cells in the next area with much larger receptive field sizes. The different relative receptive field sizes have been measured experimentally and the values range from $1: 5$ up to $1: 10$ $[28,38]$. In the model simulations we typically used a parameterization at the lower size range, namely, 1:5 for V1:MT filter sizes. Motion contrasts can be detected by mechanisms utilizing a center-surround region, for example, with opposite direction selectivity. Such opponent-velocity selective motion sensitive cells have been reported to occur in area MT as well as in the ventral division of area MST, MSTv [29]. The mechanisms of feedforward filtering and signal enhancement, modulatory feedback signal processing, and activity normalization will be discussed as follows.

3.2. Local Motion Estimation. The input processing stage for initial motion detection is divided into two steps. The first concerns cells selective to static oriented contrasts at different spatial frequencies and independent of contrast polarity to resemble model complex cells. The filtering mechanism is implemented by the following equation:

$$
\begin{aligned}
\frac{d c_{0}(\mathbf{x}, \theta, t)}{d t}= & -0.01 \cdot c_{0}(\mathbf{x}, \theta, t) \\
& +\left\{\partial_{\mathbf{x}, \theta}^{2} \Lambda_{\sigma} * I(\mathbf{x}, t)\right\}-c_{0}(\mathbf{x}, \theta, t) \\
& \cdot\left\{\Lambda_{\sigma} * \int_{\Phi}\left|\left\{\partial_{\mathbf{x}, \phi}^{2} \Lambda_{\sigma} * I(\mathbf{x}, t)\right\}\right| d \phi\right\},
\end{aligned}
$$

which is solved at equilibrium. Eight orientations $(\theta)$ were used for the simulations, " $*$ " denotes the convolution operator, $\Lambda_{\sigma}$ is a spatial weighting function (Gaussian with size parameter $\sigma$ ), and $\partial_{\mathbf{x}, \theta}^{2} \Lambda_{\sigma}$ denotes the second directional derivative along $\theta$. The response of the filtering stage is normalized by responses in a spatial neighbourhood to yield contrast dependent activity $c_{0}$. The normalization is computed by integrating the contrast responses over all orientations $\phi$ (over the domain $\Phi$ ).

The second stage considers direction-selective cells, to compute motion energy from spatiotemporal correlations for opposite motions between two consecutive image frames. 


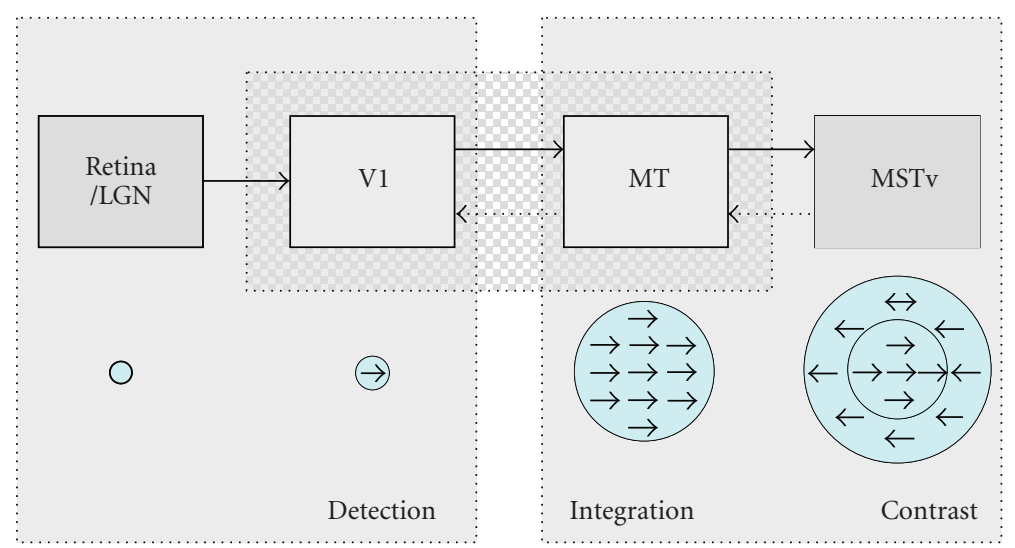

FIGURE 5: Box-and-arrow representation presenting an overview of neural connection and interaction scheme based on different cortical areas. Input images are fed forward from LGN into model area V1, where they undergo a filtering with a bank of orientation selective filters to extract local structure in an image frame. Performing a spatiotemporal correlation with these local response energies generates an initial motion signal which is forwarded to model area MT. In area MT a population code is generated to encode motion speed and direction. This integrated motion signal is further delivered to model area MSTv that may detect discontinuities in the flow field of motion vectors. The modelling framework presented here focuses on the interactive processing of motion information at the level of areas V1 and MT. We have highlighted this by the dashed grey box in the center of the figure. See text for further details.

Local motion is measured by testing a range of distinct velocities at each location, denoted by shifts $\Delta \mathbf{x}=(\Delta x, \Delta y)$ around $\mathbf{x}$ in the subsequent image frame, using properly tuned modified elaborated Reichardt detectors (ERDs; similar to [39]). (Spatial bandpass filtering of the input images to generate $c_{0}$ responses reduces spatial aliasing effects. Sampling along the temporal axis using only two consecutive frames may introduce temporal aliasing which could be prevented by temporal smoothing. In our experiments using synthetic as well as realistic test sequences we did not observe any harmful aliasing effects such that we utilized the simple approach here.) The resulting activity is denoted by $c_{1}$ :

$$
\begin{aligned}
c_{1}^{(\rightarrow)} & (\mathbf{x}, \Delta \mathbf{x}, t) \\
& =\left\{\Lambda_{\sigma} * \int_{\Phi} c_{0}(\mathbf{x}, \phi, t) \cdot c_{0}(\mathbf{x}+\Delta \mathbf{x}, \phi, t+1) d \phi\right\} \\
c_{1}^{(-)} & (\mathbf{x}, \Delta \mathbf{x}, t) \\
& =\left\{\Lambda_{\sigma} * \int_{\Phi} c_{0}(\mathbf{x}+\Delta \mathbf{x}, \phi, t) \cdot c_{0}(\mathbf{x}, \phi, t+1) d \phi\right\},
\end{aligned}
$$

pooling over all orientation-selective cells at different time steps. The final output motion response $c_{1}$ is calculated to build a population code of directional responses utilizing opponent subtractive and shunting inhibition, namely,

$$
\begin{aligned}
& \frac{d c_{2}^{(\rightarrow)}(\mathbf{x}, \Delta \mathbf{x}, t)}{d t} \\
& =-c_{2}^{(\rightarrow)}(\mathbf{x}, \Delta \mathbf{x}, t)+\left[c_{1}^{(\rightarrow)}(\mathbf{x}, \Delta \mathbf{x}, t)\right]_{+} \\
& \quad-\left(0.5+c_{2}^{(\rightarrow)}(\mathbf{x}, \Delta \mathbf{x}, t)\right) \cdot\left[c_{1}^{(-)}(\mathbf{x}, \Delta \mathbf{x}, t)\right]_{+},
\end{aligned}
$$

and the corresponding response for the opposite direction $c_{2}^{(-)}(\mathbf{x}, \Delta \mathbf{x}, t)$, both of which were solved at equilibrium. The operator $[x]_{+}=\max (x, 0)$ denotes half-wave rectification. The resulting activities $c_{2}^{(\bullet)}(\mathbf{x}, \Delta \mathbf{x}, t)$ for different velocities (encoded by $\Delta \mathbf{x}$ ) at different locations ( $\mathbf{x}$ ) indicate unambiguous motion at corners and line endings, ambiguous motion along contrasts, and no motion for homogeneous regions. The rectified activities generate positive feeding input for the subsequent motion processing stage as sketched below.

\subsection{Motion Detection and Feedforward/Feedback Processing} in Model Area V1. The core components of the model highlighted in Figure 5 are model areas V1 and MT. Once again, each model area is defined by a three-level cascade of processing steps as outlined in Figure 2 In particular, we define the response properties for model area V1 as follows. The initial filtering stage is fed by the initial motion detection as outlined above. Thus this step is governed by the simple linear processing:

$$
\tau \frac{d y_{0}^{\mathrm{V} 1}(\mathbf{x}, \mathbf{v}, t)}{d t}=-\alpha^{\mathrm{V} 1} \cdot y_{0}^{\mathrm{V} 1}(\mathbf{x}, \mathbf{v}, t)+\beta_{0}^{\mathrm{V} 1} \cdot f^{\mathrm{V} 1}\left(c_{2}(\mathbf{x}, \mathbf{v}, t)\right),
$$

with the first term $-\alpha^{\mathrm{V} 1} \cdot y_{1}^{\mathrm{V} 1}(\mathbf{x}, \mathbf{v}, t)$ denoting the activity decay with rate $\alpha^{\mathrm{V} 1}$ when driving input has been switched off, $\beta_{0}^{\mathrm{V} 1}$ is a scaling constant, and $f^{\mathrm{V} 1}(x)=x^{2}$ defines a nonlinear signal enhancement for the initial motion detection stage. The velocity code $\mathbf{v}$ is generated from the offset $\Delta \mathbf{x}$ and the directional coding denoted by " $\rightarrow$ " and “ $\leftarrow$ " in the previous stage of initial spatiotemporal correlation. These initial motion responses define the feeding input to the stage of model V1. This activity is subsequently enhanced by feedback signals delivered by neurons from higher-order stages, such as area MT in our case. As outlined above, we propose a modulating enhancement, or soft-gating, mechanism that enhances feeding inputs when corresponding 
feedback activity is available. The signal enhancement stage reads

$$
\begin{aligned}
\tau \frac{d y_{1}^{\mathrm{V} 1}(\mathbf{x}, \mathbf{v}, t)}{d t}= & -\alpha^{\mathrm{V} 1} \cdot y_{1}^{\mathrm{V} 1}(\mathbf{x}, \mathbf{v}, t)+\beta_{1}^{\mathrm{V} 1}\left(1-y_{1}^{\mathrm{V} 1}(\mathbf{x}, \mathbf{v}, t)\right) \\
& \cdot y_{0}^{\mathrm{V} 1}(\mathbf{x}, \mathbf{v}, t) \cdot\left(1+\kappa_{\mathrm{FB}}^{\mathrm{V} 1} \cdot y_{3}^{\mathrm{MT}}(\mathbf{x}, \mathbf{v}, t)\right) .
\end{aligned}
$$

The r.h.s. of this equation is composed of components that realize the modulatory enhancement of activities in a dynamic equation. Again, the first term $-\alpha^{\mathrm{V} 1} \cdot y_{1}^{\mathrm{V} 1}(\mathbf{x}, \mathbf{v}, t)$ denotes the activity decay. The second term is composed of three multiplicative components. Here, the term $\beta_{1}^{\mathrm{V} 1}(1-$ $\left.y_{1}^{\mathrm{Vl}}(\mathbf{x}, \mathbf{v}, t)\right)$ regulates the saturation of the model cell membrane (compare with the excitatory membrane conductance in $(2))$. The term $y_{0}^{\mathrm{V} 1}(\mathbf{x}, \mathbf{v}, t) \cdot\left(1+\kappa_{\mathrm{FB}}^{\mathrm{V} 1} \cdot y_{3}^{\mathrm{MT}}(\mathbf{x}, \mathbf{v}, t)\right)$ realizes the modulatory signal enhancement, or linking, mechanism as discussed in the previous section. Referring to the table in step 2 of the cascade as depicted in Figure 2 we can observe the logic of this linking mechanism. Feeding input activation, $y_{0}^{\mathrm{V} 1}(\mathbf{x}, \mathbf{v}, t)$, is required to generate a nonzero output. In other words, $y_{0}^{\mathrm{V} 1}$ gates the feedback activation that is generated by a higher-level stage of processing. The feedback signal itself consists of a tonic input level that is superimposed by the activity, $y_{3}^{\mathrm{MT}}(\mathbf{x}, \mathbf{v}, t)$, that is delivered by the output stage of model MT (see the following). The feedback activation is amplified by a constant denoted by $\kappa_{\mathrm{FB}}^{\mathrm{Vl}}$.

The final, or output, stage of the cascade is defined by a center-surround mechanism as discussed in the previous section. We suggest a generic stage of competition that can be parameterized properly in order to study the influence of different model mechanisms. The activity at the competitive stage reads

$$
\begin{aligned}
\tau \frac{d y_{2}^{\mathrm{V} 1}(\mathbf{x}, \mathbf{v}, t)}{d t} \\
=-\alpha^{\mathrm{V} 1} \cdot y_{2}^{\mathrm{V} 1}(\mathbf{x}, \mathbf{v}, t)+\left(\beta_{2}^{\mathrm{V} 1}-\delta_{2}^{\mathrm{V} 1} \cdot y_{2}^{\mathrm{V} 1}(\mathbf{x}, \mathbf{v}, t)\right) \\
\cdot y_{1}^{\mathrm{V} 1}(\mathbf{x}, \mathbf{v}, t)-\left(\lambda_{2}^{\mathrm{V} 1}+y_{2}^{\mathrm{V} 1}(\mathbf{x}, \mathbf{v}, t)\right) \\
\cdot\left\{\Lambda_{\sigma}^{\mathrm{V} 1 \text {,surr }} \stackrel{\mathbf{x}}{*} \int_{\text {vel }} y_{1}^{\mathrm{V} 1}\left(\mathbf{x}, \mathbf{v}^{\prime}, t\right) d \mathbf{v}^{\prime}\right\} \\
-\delta_{2}^{\mathrm{V} 1} \cdot y_{2}^{\mathrm{V} 1}(\mathbf{x}, \mathbf{v}, t) \\
\cdot\left\{\Lambda_{\sigma}^{\mathrm{V} 1, \text { pool }} \stackrel{\mathbf{x}}{*} \int_{\text {vel }} y_{2}^{\mathrm{V} 1}\left(\mathbf{x}, \mathbf{v}^{\prime}, t\right) d \mathbf{v}^{\prime}\right\} .
\end{aligned}
$$

The r.h.s. of this equation is again composed by several components to realize the center-surround competition corresponding to the sketch of the biophysical membrane equation depicted in Figure 3. Again, as in the previous equations, the first term $-\alpha^{\mathrm{V} 1} \cdot y_{2}^{\mathrm{V} 1}(\mathbf{x}, \mathbf{v}, t)$ denotes the rate of passive activity decay. The next two terms specify the feedforward on-center/off-surround mechanism driven by the activity from the previous stage in the hierarchy. In particular, we get $+\left(\beta_{2}^{\mathrm{V1}}-\delta_{2}^{\mathrm{V} 1} \cdot y_{2}^{\mathrm{V} 1}(\mathbf{x}, \mathbf{v}, t)\right) \cdot y_{1}^{\mathrm{V} 1}(\mathbf{x}, \mathbf{v}, t)-$ $\left(\lambda_{2}^{\mathrm{V} 1}+y_{2}^{\mathrm{V} 1}(\mathbf{x}, \mathbf{v}, t)\right) \cdot\left\{\Lambda_{\sigma}^{\mathrm{V} 1 \text { surr }} \stackrel{*}{*} \int_{\mathrm{vel}} y_{1}^{\mathrm{V} 1}\left(\mathbf{x}, \mathbf{v}^{\prime}, t\right) d \mathbf{v}^{\prime}\right\}$, with $\Lambda_{\sigma}^{\text {surr }}$ that denotes the spatial weighting kernel for the surround inhibition (the kernel is parameterized by a scaling constant $\sigma)$. The terms in brackets, namely, $\left(\beta_{2}^{\mathrm{V} 1}-\delta_{2}^{\mathrm{V} 1}\right.$. $\left.y_{2}^{\mathrm{V} 1}(\mathbf{x}, \mathbf{v}, t)\right)$ and $\left(\lambda_{2}^{\mathrm{V} 1}+y_{2}^{\mathrm{V} 1}(\mathbf{x}, \mathbf{v}, t)\right)$, denote the membrane properties for the excitatory and inhibitory driving inputs, respectively. The parameters $\beta_{2}^{\mathrm{V} 1}, \delta_{2}^{\mathrm{V} 1}$, and $\lambda_{2}^{\mathrm{V} 1}$ control the different types of center-surround interaction. For example, $\delta_{2}^{\mathrm{V} 1}=0$ will drive the center term by a purely additive input (scaled by $\beta_{2}^{\mathrm{V} 1}$ ). The constant $\lambda_{2}^{\mathrm{V} 1}$, in turn, controls whether the inhibition has a subtractive influence on the center. The multiplicative term $y_{2}^{\mathrm{V1}}(\mathbf{x}, \mathbf{v}, t)$, again, constitutes the divisive influence of the surround inhibition which is determined by the weighted integration of the activities in velocity space at each spatial location over a circular neighbourhood in the space-domain. In addition, the last inhibitory term $\delta_{2}^{\mathrm{V} 1} \cdot y_{2}^{\mathrm{Vl}}(\mathbf{x}, \mathbf{v}, t) \cdot\left\{\Lambda_{\sigma}^{\mathrm{V} 1 \text {,pool }} \stackrel{\mathrm{x}}{*} \int_{\mathrm{vel}} y_{2}^{\mathrm{V} 1}\left(\mathbf{x}, \mathbf{v}^{\prime}, t\right) d \mathbf{v}^{\prime}\right\}$ determines the integration of neuronal activations $y_{2}^{\mathrm{V} 1}(\mathbf{x}, \mathbf{v}, t)$ from the pool of cells in the output stage of model V1 in the neighbourhood of the target cell and over all velocities. Here, the kernel $\Lambda_{\sigma}^{\mathrm{V} 1, \mathrm{pool}}$ determines the spatial weighting kernel for the pooling region. The spatial neighbourhood of the pool of neurons is thought to be much larger than those of the surround of the feeding inputs (compare [36]), such that the parameterization fulfils $\sigma_{\mathrm{V} 1 \text {,pool }} \gg \sigma_{\mathrm{V} 1 \text {,surr }}$. Please note that in the final stage of competitive interaction and activity normalization the dynamical competition has been lumped into one equation and, thus, simplifies the mechanism outlined in (7). In order to do so, we assume that the integration from pooling the cell activations leads to a quick response, such that the separate components of (7) can be combined into one.

It should be further noted here that the separate equations to denote the individual stages of the processing hierarchy can be combined to yield a reduced description of the system of equations. For example, if we assume that the responses of the initial stages of filtering and feedback modulation quickly equilibrate, then both equations can be fused into one to yield

$$
\begin{aligned}
\tau \frac{d y_{1}^{\mathrm{V} 1}(\mathbf{x}, \mathbf{v}, t)}{d t}= & -\alpha^{\mathrm{V} 1} \cdot y_{1}^{\mathrm{V} 1}(\mathbf{x}, \mathbf{v}, t) \\
& +\beta_{1}^{\mathrm{V} 1}\left(1-y_{1}^{\mathrm{V} 1}(\mathbf{x}, \mathbf{v}, t)\right) \cdot f^{\mathrm{V} 1}\left(c_{2}^{(\bullet)}(\mathbf{x}, \mathbf{v}, t)\right) \\
& \cdot\left(1+\kappa_{\mathrm{FB}}^{\mathrm{V} 1} \cdot y_{3}^{\mathrm{MT}}(\mathbf{x}, \mathbf{v}, t)\right)
\end{aligned}
$$

assuming proper rescaling and adjustment of constants. Furthermore, under the assumption of quick equilibration of activities, the activity for $y_{1}^{\mathrm{V} 1}(\mathbf{x}, \mathbf{v}, t)$ can be directly plugged into the equation that denotes the final competitive stage for center-surround normalization. In sum, by simplifiying over details in the exact dynamic behavior the computational simulation of the familiy of equations can be rather simplified in order to speed up processing and to simplify the analysis of the response properties of the layered architecture of mutually coupled neuronal sheets of model neurons. In order to prevent any negative activation levels $y_{2}$ responses are half-wave rectified before they are fed forward to model area MT cells. 
3.4. Motion Integration in Model Area MT. As already pointed out in the previous section, we propose that each model area is composed of essentially the same three-level cascade of computational stages. The function of the input changes in accordance with the desired functionality of the stage of processing. Thus, filter functions, sampling rates, and individual parameterization of the individual stages change properly. Other than that, the structure of processing along the individual stages, therefore, looks almost similar in model area MT. We outline the stages in a step-by-step fashion.

The initial filtering stage is fed by the output of model area V1 and integrates over a larger spatial neighbourhood a range of different velocities. This processing step is governed by the following equation:

$$
\begin{aligned}
\tau \frac{d y_{0}^{\mathrm{MT}}(\mathbf{x}, \mathbf{v}, t)}{d t}= & -\alpha^{\mathrm{MT}} \cdot y_{0}^{\mathrm{MT}}(\mathbf{x}, \mathbf{v}, t) \\
& +\left(1-\beta_{0}^{\mathrm{MT}} \cdot y_{0}^{\mathrm{MT}}(\mathbf{x}, \mathbf{v}, t)\right) \\
& \cdot f^{\mathrm{MT}}\left(\left\{\Lambda_{\sigma}^{\mathrm{MT}} \stackrel{\mathbf{x}, \text { vel }}{*} y_{2}^{\mathrm{V} 1}(\mathbf{x}, \mathbf{v}, t)\right\}\right) .
\end{aligned}
$$

The first term of the r.h.s. of this equation, $-\alpha^{\mathrm{MT}}$. $y_{0}^{\mathrm{MT}}(\mathbf{x}, \mathbf{v}, t)$ denotes the rate of passive activity decay. The second term, like in model V1, denotes the activity integration that is modulated by the activity, $\left(1-\beta_{0}^{\mathrm{MT}} \cdot y_{0}^{\mathrm{MT}}(\mathbf{x}, \mathbf{v}, t)\right)$. The feeding input activity for the velocity selective target cell is integrated over a space-velocity neighbourhood as depicted by $\left\{\Lambda_{\sigma}^{\mathrm{MT}} \stackrel{\mathbf{x}, \text { vel }}{*} y_{2}^{\mathrm{V} 1}(\mathbf{x}, \mathbf{v}, t)\right\}$. The function $f^{\mathrm{MT}}(x)$, again, is used to nonlinearly transform the input signal by, for example, a squaring operation. The second stage again implements a modulating enhancement mechanism that enhances feeding inputs by feedback signals. This reads

$$
\begin{aligned}
\tau \frac{d y_{1}^{\mathrm{MT}}(\mathbf{x}, \mathbf{v}, t)}{d t}= & -\alpha^{\mathrm{MT}} \cdot y_{1}^{\mathrm{MT}}(\mathbf{x}, \mathbf{v}, t) \\
& +\left(1-\beta_{1}^{\mathrm{MT}} \cdot y_{1}^{\mathrm{MT}}(\mathbf{x}, \mathbf{v}, t)\right) \cdot y_{0}^{\mathrm{MT}}(\mathbf{x}, \mathbf{v}, t) \\
& \cdot\left(1+\kappa_{\mathrm{FB}}^{\mathrm{MT}} \cdot y_{3}^{\mathrm{high}}(\mathbf{x}, \mathbf{v}, t)\right)
\end{aligned}
$$

Again, the first term of the r.h.s. of this equation $-\alpha^{\mathrm{MT}}$. $y_{1}^{\mathrm{MT}}(\mathbf{x}, \mathbf{v}, t)$ denotes the rate of activity decay. The second term is composed of three multiplicative components, like in the equation for model V1, with $\left(1-\beta_{1}^{\mathrm{MT}} \cdot y_{1}^{\mathrm{MT}}(\mathbf{x}, \mathbf{v}, t)\right)$ to regulate the saturation property of the model cell membrane. If one wishes to linearly integrate the integrated filter responses, the shunting term can be eliminated by setting $\beta_{1}^{\mathrm{MT}}=0$. The term $y_{0}^{\mathrm{MT}}(\mathbf{x}, \mathbf{v}, t) \cdot\left(1+\kappa_{\mathrm{FB}}^{\mathrm{MT}} \cdot y_{3}^{\text {high }}(\mathbf{x}, \mathbf{v}, t)\right)$ allows further modulatory input from other stages in the visual hierarchy of processing. For example, as outlined in Figure 5, input can be incorporated that computes the presence of motion discontinuities and these signals can be utilized to enhance the representation of motion at the stage of model MT (compare [37]). Also, attention signals can be incorporated to bias the competition at the output stage (compare [40]). In this case, either spatial attention signals may be incorporated that enhance the activities at given spatial locations, or, feature attention signals may enhance the presence of specific features irrespective of their location. In the computational framework presented here, we assume no modulating input from any higher-order stages, such that $\kappa_{\mathrm{FB}}^{\mathrm{MT}}=0$. As a consequence, the bottom-up feeding input is simply fed forward without major changes, namely,

$$
\begin{aligned}
\tau \frac{d y_{1}^{\mathrm{MT}}(\mathbf{x}, \mathbf{v}, t)}{d t}= & -\alpha^{\mathrm{MT}} \cdot y_{1}^{\mathrm{MT}}(\mathbf{x}, \mathbf{v}, t) \\
& +\left(1-\beta_{1}^{\mathrm{MT}} \cdot y_{1}^{\mathrm{MT}}(\mathbf{x}, \mathbf{v}, t)\right) \cdot y_{0}^{\mathrm{MT}}(\mathbf{x}, \mathbf{v}, t) .
\end{aligned}
$$

For parameter settings of $\alpha^{\mathrm{MT}}=1$ and $\beta_{1}^{\mathrm{MT}}=0$ the equation reduces to an identity transform of the input activations $y_{0}^{\mathrm{MT}}(\mathbf{x}, \mathbf{v}, t)$. Finally, the output stage of the cascade is again defined by a center-surround mechanism of the same generic structure as above. The activity at the competitive stage reads

$$
\begin{aligned}
\tau \frac{d y_{2}^{\mathrm{MT}}(\mathbf{x}, \mathbf{v}, t)}{d t}= & -\alpha^{\mathrm{MT}} \cdot y_{2}^{\mathrm{MT}}(\mathbf{x}, \mathbf{v}, t) \\
& +\left(\beta_{2}^{\mathrm{MT}}-\delta_{2}^{\mathrm{MT}} \cdot y_{2}^{\mathrm{MT}}(\mathbf{x}, \mathbf{v}, t)\right) \\
& \cdot y_{1}^{\mathrm{MT}}(\mathbf{x}, \mathbf{v}, t)-\left(\lambda_{2}^{\mathrm{MT}}+y_{2}^{\mathrm{MT}}(\mathbf{x}, \mathbf{v}, t)\right) \\
& \cdot\left\{\Lambda_{\sigma}^{\mathrm{MT}, \text { surr }} \stackrel{\mathbf{x}}{*} \int_{\text {vel }} y_{1}^{\mathrm{MT}}(\mathbf{x}, \mathbf{v}, t) d \mathbf{v}\right\} \\
& -\delta_{2}^{\mathrm{MT}} \cdot y_{2}^{\mathrm{MT}}(\mathbf{x}, \mathbf{v}, t) \\
& \cdot\left\{\Lambda_{\sigma}^{\mathrm{MT}, \mathrm{pool}} \underset{*}{\mathbf{x}} \int_{\mathrm{vel}} y_{2}^{\mathrm{MT}}(\mathbf{x}, \mathbf{v}, t) d \mathbf{v}\right\} .
\end{aligned}
$$

The r.h.s. of this equation realizes the center-surround competition that considers the surround inhibition for the feeding input as well as the normalization by the pool of neurons in the same layer. The first term $-\alpha^{\mathrm{MT}} \cdot y_{2}^{\mathrm{MT}}(\mathbf{x}, \mathbf{v}, t)$ denotes the rate of passive activity decay. The next two terms specify the feedforward on-center/off-surround mechanism driven by the feeding input activation from the previous processing stage in model MT, namely, $y_{1}^{\mathrm{MT}}(\mathbf{x}, \mathbf{v}, t)$ for the center activity and $\left\{\Lambda_{\sigma}^{\mathrm{MT} \text {,surr }} \stackrel{*}{*} \int_{\mathrm{vel}} y_{1}^{\mathrm{MT}}(\mathbf{x}, \mathbf{v}, t) d \mathbf{v}\right\}$ for the surround. Both input components serve as variable conductance excitatory and inhibitory input, respectively, which are modulated by the leading terms in brackets. The symbol $\Lambda_{\sigma}^{\mathrm{MT} \text {,surr }}$ denotes the spatial weighting kernel for the surround inhibition in model area MT. Again, the parameters $\beta_{2}^{\mathrm{MT}}, \delta_{2}^{\mathrm{MT}}$, and $\lambda_{2}^{\mathrm{MT}}$ control the different types of center-surround interaction. For example, $\delta_{2}^{\mathrm{MT}}=0$ will drive the center term by a purely additive input (scaled by $\beta_{2}^{\mathrm{MT}}$ ). The constant $\lambda_{2}^{\mathrm{MT}}$, in turn, controls whether the inhibition has a subtractive influence on the center, and the multiplicative term $y_{2}^{\mathrm{MT}}(\mathbf{x}, \mathbf{v}, t)$, again, defines the divisive influence of the surround inhibition (from weighted integration of activities in velocity space over a circular spatial 
neighbourhood). In addition, the inhibitory term $\delta_{2}^{\mathrm{MT}}$.

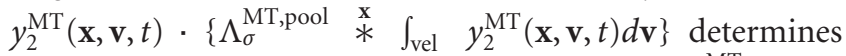
the integrated activities over a pool of cells of $y_{2}^{\mathrm{MT}}(\mathbf{x}, \mathbf{v}, t)$ neurons. The kernel $\Lambda_{\sigma}^{\mathrm{MT} \text {,pool }}$ defines the spatial weighting kernel for the pooling region which is much larger than the surround kernel for the feeding inputs, such that $\sigma_{\mathrm{MT} \text {,pool }} \gg$ $\sigma_{\mathrm{MT} \text {,surr }}$ holds.

A similar consideration as for modelling V1 responses also applies to model MT cell responses. As already pointed out above, we do not consider any modulatory input to model MT cells which leads to an identity stage of processing, given proper parameter adjustments. Since the initial stage of filtering at the input to the MT cascade integrates over spatial position and velocities of the V1 motion detection input, this step can also be directly summarized into the last equation. As a consequence, the dynamic MT processing can be formulated by one equation that defines the MT activity, namely,

$$
\begin{aligned}
& \tau \frac{d y_{2}^{\mathrm{MT}}(\mathbf{x}, \mathbf{v}, t)}{d t} \\
& =-y_{2}^{\mathrm{MT}}(\mathbf{x}, \mathbf{v}, t)+\left(\beta^{\mathrm{MT}}-y_{2}^{\mathrm{MT}}(\mathbf{x}, \mathbf{v}, t)\right) \\
& \cdot f^{\mathrm{MT}}\left(\left\{\Lambda_{\sigma}^{\mathrm{MT}} \stackrel{\mathbf{x}, \text { vel }}{*} y_{2}^{V 1}(\mathbf{x}, \mathbf{v}, t)\right\}\right)-\left(\lambda^{\mathrm{MT}}+y_{2}^{\mathrm{MT}}(\mathbf{x}, \mathbf{v}, t)\right) \\
& \cdot\left\{\Lambda_{\sigma}^{\mathrm{MT}, \text { surr }} \stackrel{\underset{*}{*}}{*} \int_{\text {vel }} f^{\mathrm{MT}}\left(\left\{\Lambda_{\sigma}^{\mathrm{MT}} \stackrel{\mathbf{x}, \text { vel }}{*} y_{2}^{V 1}(\mathbf{x}, \mathbf{v}, t)\right\}\right)\right\} \\
& -y_{2}^{\mathrm{MT}}(\mathbf{x}, \mathbf{v}, t) \cdot\left\{\Lambda_{\sigma}^{\mathrm{MT}, \text { pool }} \stackrel{\mathbf{x}}{*} \int_{\text {vel }} y_{2}^{\mathrm{MT}}(\mathbf{x}, \mathbf{v}, t) d \mathbf{v}\right\},
\end{aligned}
$$

utilizing here parameter settings $\alpha^{\mathrm{MT}}=\delta_{2}^{\mathrm{MT}}=1$. (The summarized activity in model MT is expressed by one equation by lumping the individual stages of the cascade. In order to keep the nomenclature used so far we choose to assign the response level to the output of the model area. Thus the resulting activity is indexed with the final level corresponding to $y_{2}$.) In this combined equation (20) saturation levels $\beta^{\mathrm{MT}}$ and $\lambda^{\mathrm{MT}}$ occur that have the same computational roles as in the separate equations (see Section 2 for the general description). In order to avoid confusion we omitted indices here.

These model equations in the simplified form were subsequently used to simulate the motion responses to various input sequences. In order to emphasize the explanatory power of the approach to explain biological information processing, we demonstrate how the model can cope with input that were used in various experimental settings in animal studies (neurophysiology) and human behavioural investigations (psychophysics). In order to demonstrate the potential of the approach to deal with realistic input sequences from various technical application domains, we also show results for selected benchmark test sequences and data that have been acquired in an application-oriented project scenario.

\section{Simulation Results}

In this section we present results of computational investigations using the model framework as outlined above. The results are grouped to first demonstrate the capability of the model to explain experimental findings from perceptual psychophysics and physiology. In the second part we show several results for realistic image sequences from benchmark data repositories and data related to application projects.

Before presenting the details of the simulation results we summarize few details that are common to all computational experiments, such as the parameterization of the computational stages and the display of results. The extended Reichardt detector scheme as outlined in Section 3.1 has been utilized in all experiments for initial motion estimation. The initial responses are transferred through a square nonlinearity $\mathrm{f}(\cdot)$ to generate $y_{0}^{V 1}(\mathbf{x}, \mathbf{v}, t)$. The feedforward centersurround mechanisms at the stages of model area V1 and MT to generate $y_{2}^{V 1}(\mathbf{x}, \mathbf{v}, t)$ and $y_{2}^{\mathrm{MT}}(\mathbf{x}, \mathbf{v}, t)$ activities, respectively, utilize a small component for subtractive inhibition: $\lambda_{2}^{V 1}>0, \lambda_{2}^{\mathrm{MT}}>0$. All experiments, except for the comparison study shown in Figures 8 and 9, only use feedforward surround inhibition, thus $\delta_{2}^{\mathrm{MT}}=0$. In the case shown in Figure 9 the effects of feedforward surround inhibition in the output stage of model MT are compared against the modulatory surround normalization from the pool of neurons. The results of processing are shown in a color code that has been taken from [13]. Here, the hue component encodes the direction (compare the color wheel presented as a legend in the figures) while the color saturation encodes speed. In addition to this Baker-style visualization, color transparency levels were set in accordance with confidence as computed from the overall motion energy activation calculated at each position. In addition, the flow direction is depicted with black triangles symbolizing vectors with direction and length parameterized in accordance with the local velocity. The model used a fixed set of parameter settings. These are listed in a separate table that is included in the newly incorporated appendix.

The simulations were run by using a library of $\mathrm{C}++$ software that has been developed by the authors of this paper. The implementation uses graphic card technology and the CUDA programming environment to accelerate computation of mathematical and image processing operations. In cases indicated we utilized steady-state equations as outlined in Section 3 to further speed up processing. We got a performance to process about one image frame per second with a spatial resolution of $320 \times 240$ pixels. The full dynamic equations have been numerically integrated for model variants when steady-state solutions could not be used, for example, for pooling the activities in the output stage of a model area to normalize activations. Numerical integration used Euler's one-step method.

4.1. Results for Data Sets Used in Animal and Human Experiments. In this section we have particularly focused on the processing that aims at explaining empirical results obtained in experimental studies such as in psychophysics and animal physiology. We show three example results, namely, the 


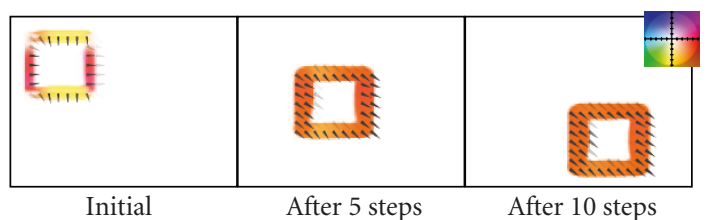

(a)

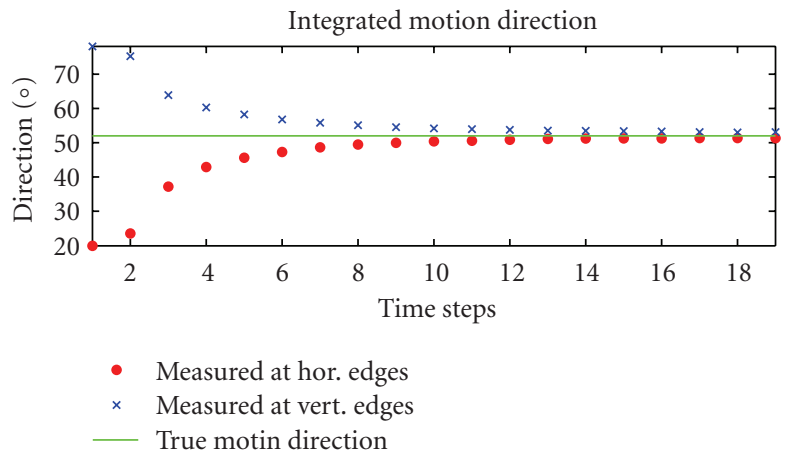

(b)

FIGURE 6: This example shows a moving square with elongated boundaries and homogeneous surface layout (image size is $320 \times 240$ pixels). Motion direction is $38.7^{\circ}$ in clockwise direction measured against the horizontal axis (down-right) at a speed of $(\Delta x, \Delta y)=$ $(4.0,5.0) \mathrm{px} /$ frame. Image frames have been lowpass filtered to avoid aliasing. Motion correspondences are tested in the range of $\Delta= \pm 7$ $\mathrm{px} /$ frame along the horizontal and vertical directions, respectively. The local average motion activity is displayed by a vector and a color code presented in the palette on the right. The true motion direction can only be measured initially (first frame) at the four corners while along the $1 \mathrm{D}$ edges only the normal flow components orthogonal to the contrasts are detected (aperture problem). Feedforward and feedback interactions between model areas V1 and MT ensure that the true object motion signals are integrated and further propagated along the outline boundary of the object to disambiguate locally ambiguous estimates (see second and third frames on the top). The initial direction error decreases over time until the true object motion is achieved at all parts of the object and a coherent motion representation has been built over time. The temporal resolution of the aperture problem has been measured in neurophysiological experiments [41] and demonstrates that MT cells adapt to the true velocity over time, just as in these simulations.

dynamic solution of the aperture problem, a hysteresis effect in the temporal update of the motion representation given contradicting evidence for motion direction, and the motion interpretation for configurations seen through an occluding window.

\subsubsection{Temporal Resolution of the Motion Aperture Problem.} Pack and Born [41] have demonstrated that monkey MT cells resolve the aperture problem over time. Initially, cells measure normal flow for extended surface outlines but signal correct velocities at localized image features, such as corners. Over time depending on the distance of the contour location from the position of localized features (along the same boundary) neurons in MT change their direction selectivity. Over a time course of approximately 80 milliseconds the peak selectivity changes to signal the true motion direction. In the computational experiment we used a square region that moved with constant speed rightwards along the downward diagonal. The motion estimates are displayed for different temporal steps showing the initial signaling of normal flow along the boundary of the square. Over time the initially correct motion estimated at the localized corners is propagated by feedforward and feedback interactions such that the uncertainty is resolved after approximately 8 time steps (results were shown for the 5th and the 10th steps, Figure 6(a)). The results and their changing direction selectivity are shown for the different edge orientations for all steps (Figure 6(b)).

4.1.2. Hysteresis Effect for Motion Interpretation. In the next experiment a cloud of random dots is moving towards the right (100\% dots moving coherently). Over time, for each frame two percent of the dots (at random positions) switch their movement direction into the opposite, namely, to the left. After 25 frames of the video sequence $50 \%$ of the dots are moving to the right while $50 \%$ are moving to the left. For even longer duration more and more dots move to the left until finally all dots are moving to the left. The challenge of the demonstration is that perceptually it takes time before suddenly the observer notices an abrupt switch in motion direction. Such displays have been observed to induce perceptual hysteresis which indicates an interaction between sensory processing and a short-term memory [42]. A simple linear motion integration mechanism, such as the spatiotemporal motion energy filters, predicts almost linear response behaviour. The response strength of a cell that is 


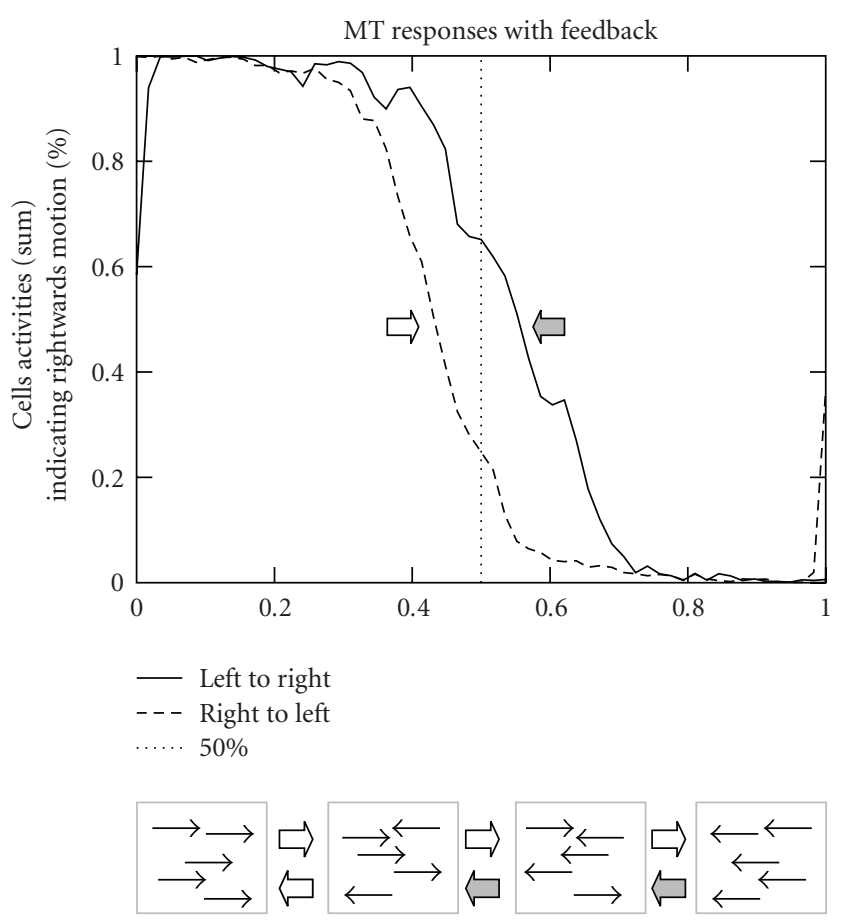

(a)

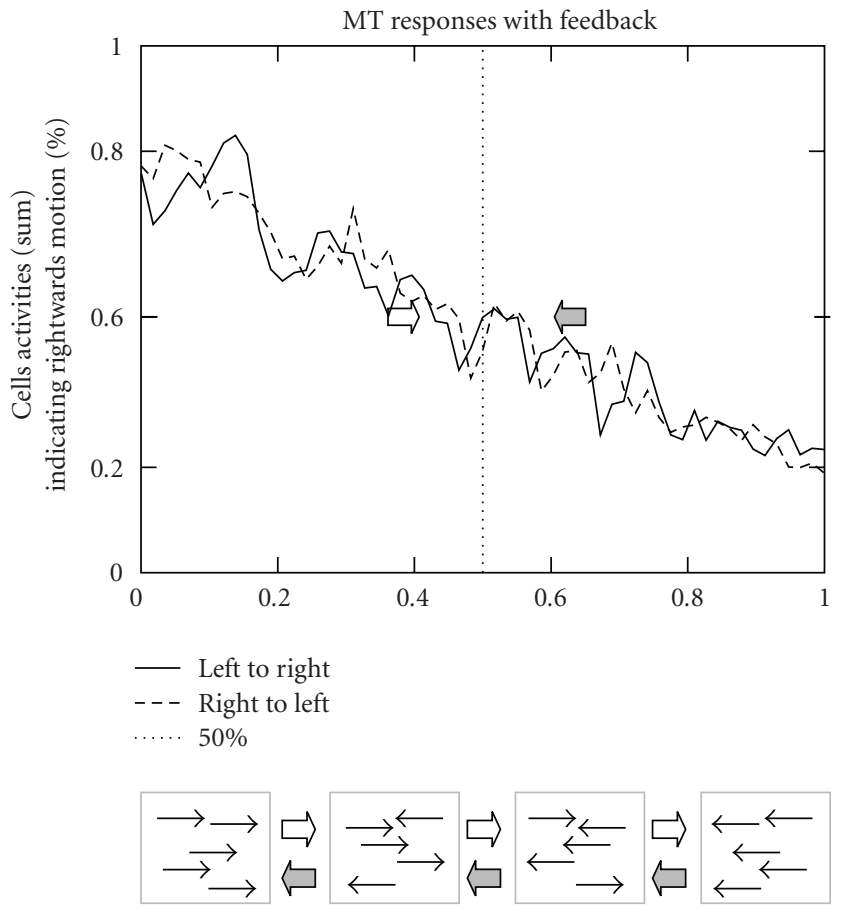

(b)

FIgURE 7: This example demonstrates the inertia of the dynamic feedforward and feedback interaction causing a perceptual hysteresis effect. The proportion of MT cell activities indicating rightward motion ( activities $_{\text {right }} / \Sigma$ activities left $)$ is plotted for each frame, processing two random dot kinematograms (the sequence shows 60 moving dots and consists of 60 frames with $40 \times 40 \mathrm{px} /$ frame). Random dots are initialized at random positions and a horizontal velocity of $3 \mathrm{px} / \mathrm{frame}$. All dots are initially moving in the same direction (in the first sequence (solid line) dots have rightward motion; in the second sequence (dashed line) dots have leftward motion). In each frame of a sequence one moving dot switches from the initial direction to now move in the opposite direction. Over time the percentage of dots still moving into the initial direction decreases linearly as the number of dots moving in the opposite direction increases in the same way. (a) Feedback processing disambiguates the signal and generates a directional hysteresis effect that indicates the inertia generated by locking in the prediction from top-down feedback of a motion direction measured over time. Initially estimated motion is slightly ambiguous (80\% correct and 20\% incorrect motion) since the correlation detectors confound local dot correspondences. This uncertainty is resolved after few iterations by model MT cells such that coherent motion is signaled. The response for the sequence that started with 60 dots moving to the right (solid line) switches the sensed motion from dominant rightward to leftward motion when $60 \%$ up to $75 \%$ of the dots have switched their initial motion direction. Thus the network responses still keep their represented motion activity beyond the condition when half of the dots move in opposite directions (in our case 30 dots moving to the right and 30 dots moving to the left). This behavior is influenced by the history of previous activities since shifting the point of perceptual decision depends on whether the sequence started with $100 \%$ rightward motion or 100\% leftward motion (hysteresis). (b) Without feedback, no hysteresis is generated. The sum of cell activities indicating rightward motion is proportional to the relative number of dots moving to the right (solid line). The initial ambiguity of $80 \%$ correct and 20\% incorrect motion is not resolved. With each frame when more and more incoherence occurs due to the dots that switch their motion direction, the estimated motion responses linearly reduce their response amplitude. This behavior is symmetric for both test cases starting with coherent dot motion to the right or to the left, respectively. As predicted in this case when half of the dots move in opposite directions, the motion signal reduced to $50 \%$ of the maximum. The figure is reprinted with permission from Bayerl and Neumann [14].

tuned to rightward motion will decline in proportion to the number of dots moving in the preferred direction. This is demonstrated for MT cells if the feedback connection to V1 cells is extinguished such that the cells merely average the input activations from motion detectors (Figure $7(\mathrm{a})$ ). When the feedback loop is closed, the MT responses show a hysteresis effect in that a cell selective to rightward motion keeps its response for quite a long period when initially probed by $100 \%$ dot motion in the preferred direction. Only when the opposite motion becomes overwhelming, the response quickly drops such that the decision is now for the opposite direction of motion (Figure 7(b)).
4.1.3. Perceptual Motion Benchmark. In a joint effort we have developed a framework for benchmarking biologically inspired motion mechanisms, similar in the spirit as proposed in the computer vision community. Briefly, we attempt to build up a repository of classic motion stimuli that have been used in studies of visual psychophysics and physiology. For those sequences resolution, luminance, and contrast levels, object speed, and stimulus size are all known and can be controlled. The general idea is that different biologically inspired models, as well as computer vision approaches that claim to incorporate biological plausible computational steps, can evaluate their models on a data set 


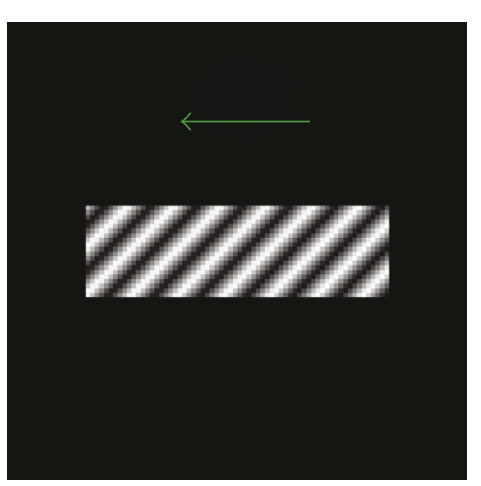

(a)

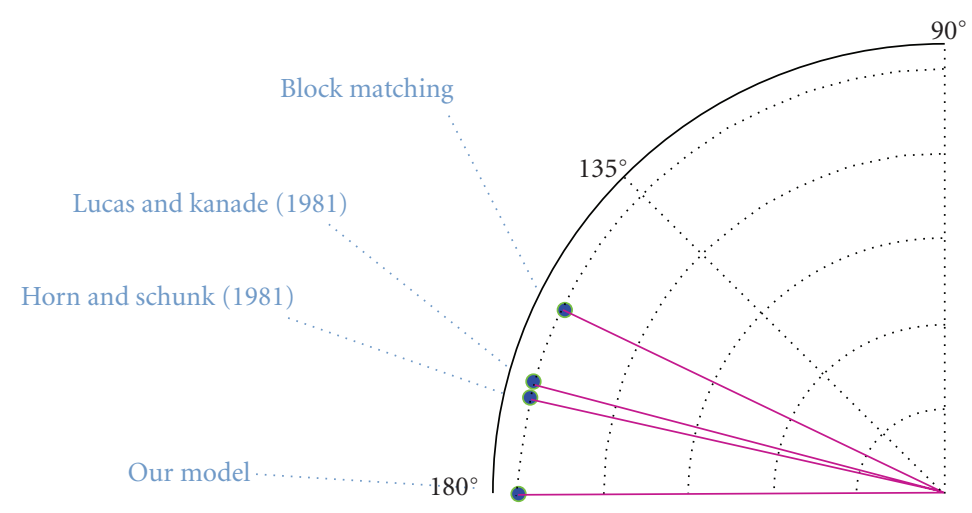

(b)

FIGURE 8: The biologically motivated mechanisms for motion processing demonstrate their capability to process test data used in perceptual experiments. The results were compared against the responses generated by computer vision algorithms that have been tuned to mathematical precision and optimality. We tested a classical block matching scheme often used in simple video encoders as well as more elaborate mechanisms for least-squares optimization of local flow (Lucas and Kanade [44]) and a regularization scheme that imposes a first-order smoothing constraint to minimize the total change in flow field gradient [2]. One example test case is the barberpole illusion (a). Humans perceive a horizontal motion for the stimulus with a moving grating behind a horizontal aperture. Classical computer vision approaches are biased in their global estimation by the normal motion direction for the extended bars. This is indicated by the upward motion at the smaller edges of the aperture which is shown by the mean velocity direction (b).

that is provided at a website and can be downloaded. It is beyond the scope of the contribution here, but we present a result for a selected sequence (the so-called barberpole illusion) that is from the benchmark repository. Details of the benchmark and website information can be found in [43]. We present the results here in order to demonstrate that the biologically inspired modelling framework leads to superior performance in comparison with computational vision methods and that the new results are comparable with human performance.

The barberpole sequence is presented for a horizontally elongated rectangular window and a luminance grating that is moving to the left and upward along the normal flow direction (see Figure 8). The challenge is that the perceptual judgement of the object motion is biased by the elongation of the window. It has been argued that the numbers of terminators moving along the horizontal direction outnumber those for the vertical direction and thus cause a bias. However, this alone cannot fully account for the percept of horizontal figure motion since a simple integration mechanism would predict a weighted average; the more extended the window is, the more the average velocity tends towards the horizontal. This is essentially what classical computer vision models compute. While the block matching achieves only a minor bias, the least square optimization approach by Lucas and Kanade [4] and the regularization approach of [2] produce similar results to get a strong bias due to the smoothness constraint that is propagated over the rectangular region. The neural model proposed here, however, is able to compute the true velocity. This is achieved through the feedforward and feedback interaction where the majority of feature flow generates a strong bias for the horizontal direction which, in turn, overwhelms the low evidence generated at line ends in favor of vertical flow (see Figure 8).
4.2. Results for Real-World Sequences. In this section we now focus on processing real-world data in order to demonstrate that the neural mechanism can also cope with test images from realistic scenarios and do not only present lab artifacts that cannot handle realistic image data. We show two example results, namely, the processing of an image sequence from the benchmark database developed by Baker et al. [13] and some results of processing different video sequences taken from surveillance test cases acquired in a soccer stadium.

4.2.1. Computer Vision Benchmark Data. Baker et al. [13] have proposed a video benchmark database with motion sequences to challenge available approaches for computer vision motion detection and integration algorithms. Here, we demonstrate the computational competency of the neural model mechanisms by showing results of processing for the RubberWhale test sequence (Figure 9(a)). For all test sequences the ground truth data is available such that the overall error of estimated flow can be evaluated. We aim here to demonstrate that the model is capable of reliably processing complex image sequences. We have computed different error measures, namely, the angular error between true and estimated flow as well as the end-point error that takes into account the contributions of speed and direction (see [13] for details). We have displayed heat maps with the different error measures in order to get a better impression how the strength and position of errors are arranged in the scene of moving objects. Also we have calculated error distributions for the estimated errors to better judge the error likelihoods and variations that occur in the test cases (see Figures 9(b) and 9(c)). We compare two simulation results where the model selectively switches between the model 


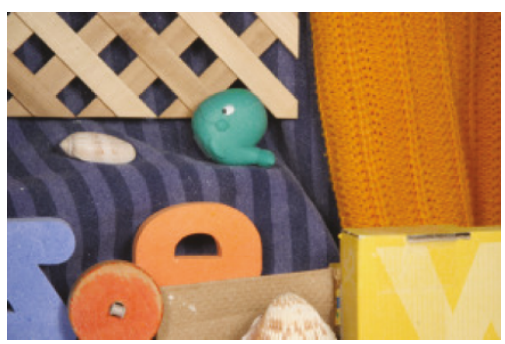

(a)
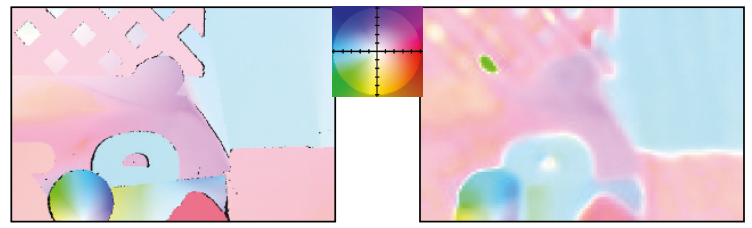

$\mathrm{AE}$
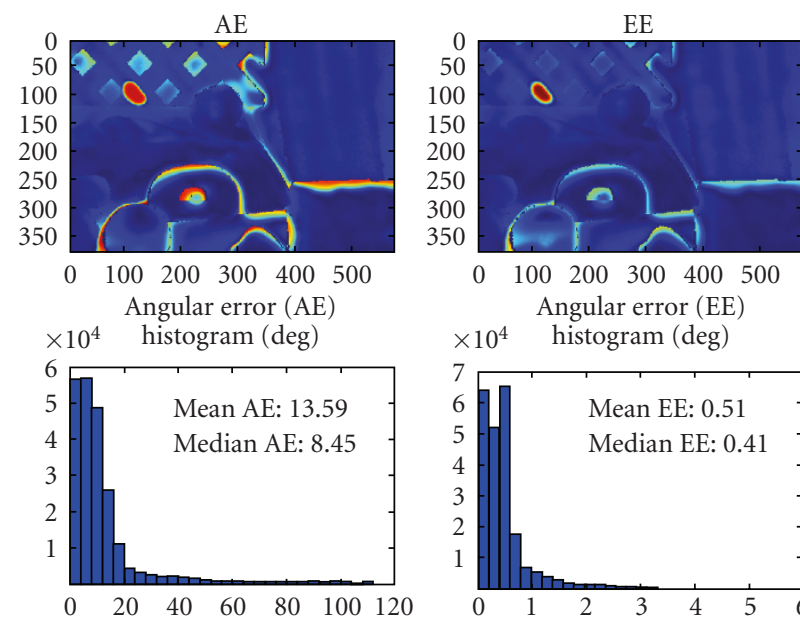

(b)

$\times 10^{4} \quad$ histogram (deg)

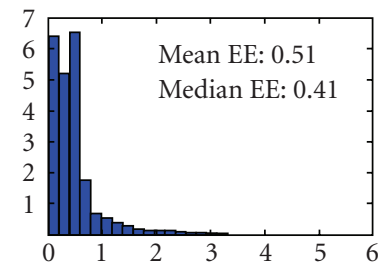

)

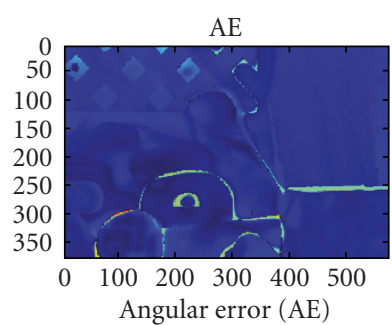

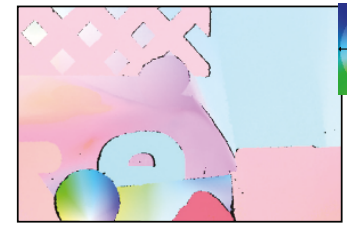
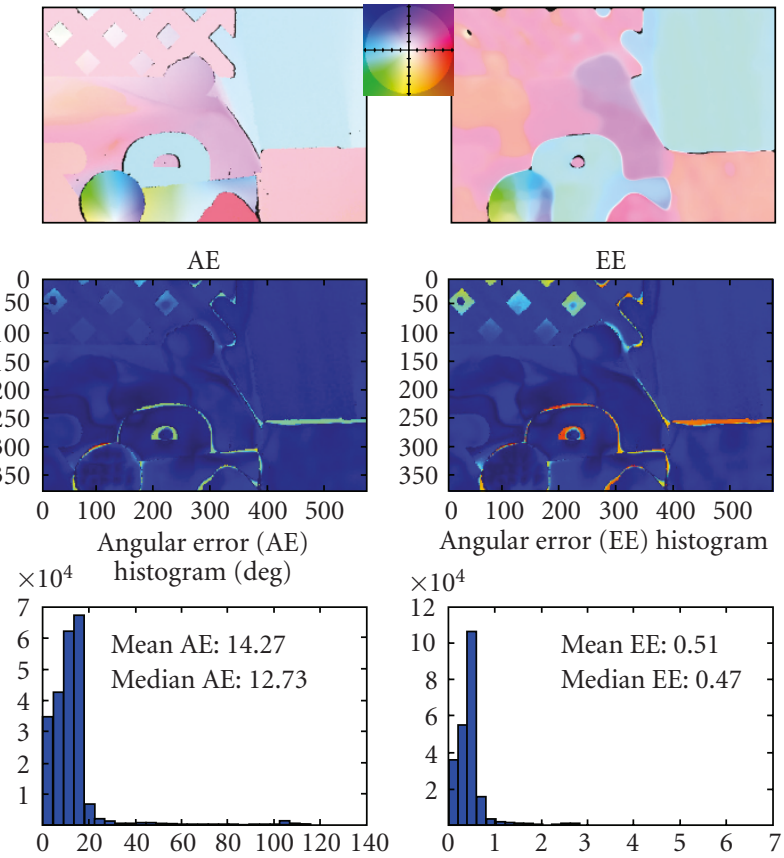

(c)

FIGURE 9: The proposed biologically inspired motion processing scheme has also been applied to realistic natural images sequences recently proposed to benchmark state-of-the-art computer vision algorithms [13]. (a) One frame of the RubberWhale sequence from the Baker et al. image sequence database. (b and c) In the top row of each subfigure, the ground truth optic flow (left) and simulation results for model MT neurons (right) are shown. The bottom row shows the angular error (AE) as well as the endpoint error (EE). The center row shows the errors as a heat map while in the bottom row the error distribution is shown. The error values shown only incorporate those pixel positions that were defined in the ground truth, and nondefined occlusion regions were excluded from the statistics. On the left (b) a variant of the model without recurrent normalization from pooling neuronal activity in area $\mathrm{MT}\left(\delta_{2}^{\mathrm{MT}}=0\right)$ is used whereas in the figures on the right (c) a parameterization with $\delta_{2}^{\mathrm{MT}}>0$ produces results with higher accuracy for homogeneous regions, while error measures increase at motion discontinuities. Considering the error distributions for the different measures in the bottom row shows that the spread is reduced for the pool normalization. However, those errors remaining deviate from zero and thus generate an overall weaker performance. These errors are focused mainly around discontinuities as indicated by the heat map representations.

components at the final stage of processing in the model areas, namely, the center-surround competition for contrast enhancement and activity normalization. In Figure 9(b) the results have been generated for feedforward center-surround competition as in the previous computational experiments, whereas in Figure 9(c) the shunting inhibition is fed by the pool of neurons in order to normalize the response at the target cell. On a first glance the model in Figure 9(c) seems to show improved performance in comparison to model variant in Figure 9(b) in terms of the overall angular and endpoint error, respectively, since the error distributions for pool normalization are greatly reduced in spread. However, a closer look at the data distribution as well as a look at the mean and median values indicates that the overall judgement must be more differentiated. Those errors that remain in the case of Figure 9 (c) peak at values which deviate from zero such that the median also has a maximum around this value. In the case of Figure 9(b) the peak occurs closer at zero so that the median (as well as the mean) values are smaller-although the error variation is larger. Considering the heat maps in Figure 9 the errors for the pool normalization are mainly localized around 


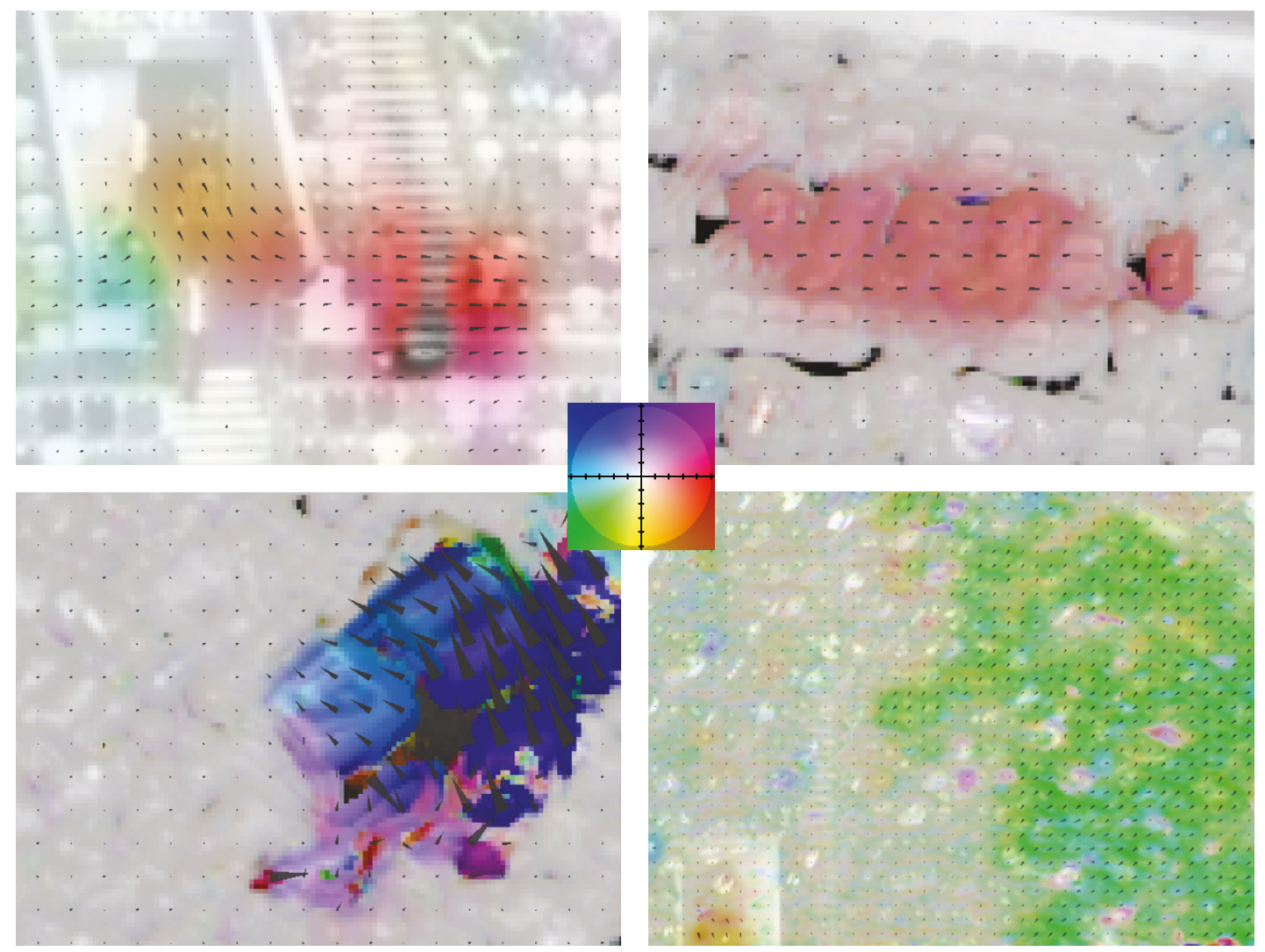

FIGURE 10: Optical flow has been estimated using the proposed neural architecture to process sample image sequences from video surveillance scenario acquired in a soccer stadium. Here, movement of single persons and groups of people is detected and integrated for different typical scenarios, such as single persons entering the stadium (top left), groups of people leaving the seating area (top right), the classification of salient events like flag waving (bottom left), and the flow estimation for a group of jumping people (bottom right). The motion is again displayed in the direction color code of the palette given in the center.

motion discontinuities generated by surface occlusions. If these regions would be excluded from the analysis, the overall performance of the model variant that utilizes the pool normalization at the output stage of the processing cascade in model MT would outperform the model variant in (b).

4.2.2. Image Sequences from Surveillance Datasets. In a recent project biologically inspired model mechanisms have been evaluated using different sequences of motion generated by crowded scenes of people. These video sequences have been acquired in a soccer stadium and show different types of motion behaviour. For these sequences there is no ground truth available. We show four different sequences and the estimated flow. The variety of image content and complex flow patterns impose a challenge since the objects (persons, flags, groups of people) were observed from a distance with varying spatial resolution. For example, the image frame in the upper left as compared to the one at the bottom right (Figure 10) shows different levels of zooming into the scene showing people at different spatial detail and resolution. The model mechanisms automatically deal with these size variations though its iterative computation and the integration of fine- and coarse-grain detail in the respective scenes.

\section{Discussion}

In this contribution, we present a computational framework of motion processing that has been derived from neuroscience data and is also capable of processing realistic image sequences. Both available key findings and the modeling framework have been briefly presented. The modeling approach has been developed in the course of the interpretation of the empirical findings from anatomy, physiology, and behavioral data. The main contributions of the modeling framework are as follows.

(i) A framework for hierarchical motion processing is proposed that consists of layered sheets of interconnected model neurons to implement generic components as building blocks for cortical model areas,

(ii) We suggest that feedforward and feedback processes interact in order to implement a hierarchically organized scheme of motion feature processing for detection and integration. Feedback signals act as reentrant modulators that can selectively enhance those feeding input activations that match the larger contextual signal representation that is built, for example, by stages higher up in the processing hierarchy. 
(iii) Reentrant mechanisms for modulatory signal enhancement together with subsequent feature competition using center-surround mechanisms for activity normalization implement a framework to bias the competition of distributed feature activities. The net effect of such combined processing in the feature processing cascade implements the selective amplification of salient features and the inhibition of responses due to clutter.

The scheme thus allows assembling more complex processing mechanisms into a network of computational building blocks by incorporating hierarchical sweeps of feature processing and modulating interactions along the reverse hierarchy of feedback processing.

In the following, we will discuss the biological plausibility of the model and give a brief assessment in comparison to other existing models of motion processing, both from biology and technical computer vision approaches.

5.1. Relevance and Biological Plausibility. There is both structural and functional evidence for the mechanisms and layered organization of our model. Anatomical and physiological studies suggest inter- and intra-areal connections also used in our model [11, 45]. Motion-sensitive cells can be found in MT as well as in V1 [46]. Physiological studies [47] have shown that cells in V1 are sensitive to component motion (motion along oriented contrasts) while cells in MT are less sensitive to oriented components but signal pattern motion during the course of their temporal activation. Recent physiological evidence suggests that V1 cells can partially encode pattern motion (thus, motion independent of the orientation). Pack et al. [48] showed that the time course of a subpopulation of V1 cells is similar to the time course of cells in MT solving the aperture problem near line endings. This is consistent with the prediction of our model that cells in V1 and MT are disambiguated simultaneously as a consequence of feedback and local competitive interaction.

The proposed modulatory feedback mechanism is supported by recent physiological investigations of feedback connections between early visual areas (V1, V2, and V3) and MT [32, 49]. For example, Hupé et al. [32] show that cell activities in V1 are highly affected by feedback from MT in an excitatory manner shortly after stimulus onset. This is consistent with our model, in which only excitatory feedback modulation is used to enhance activities by increasing their gain. As a result of recurrent processing, and consistent with physiological recordings of the time course of MT neurons [41], our model disambiguates the motion signal shortly after stimulus onset. Here, the time to establish the final percept is influenced by the strength of the feedback connections, the RF field size ratio between V1 and MT, and, as a prediction of our model, the spatial extension of the region of ambiguous motion. The time course of MT cell populations was also investigated by Pack and Born [41] for different bar lengths (2-8 degrees). Consistent with our results, the time required to disambiguate such stimuli was roughly proportional to the bar length.
5.2. Comparison with Other Models of Motion Processing. The computational mechanisms and different stages in a processing cascade as utilized by the presented model were also used in other biologically inspired as well as computer vision models.

5.2.1. Feedforward Models and Optimization Approaches. Simoncelli and Heeger [50] proposed a model of detecting motion energy in areas V1 and MT using linear spatiotemporal filters. Individual motion estimates are normalized by dividing individual responses through the average response of activity in a spatial neighborhood. Such a center-surround mechanism has also been employed in our model. We achieve such normalization of activity by an antagonistic mechanism that involves shunting inhibition for the net feeding input as well as the shunting inhibition generated by the average activity in the pool of cells surrounding the target cell. The net effect leads to a divisive inhibition at individual locations by average neuron activities integrated over a neighbourhood in the space-velocity domain. Unlike Simoncelli and Heeger, we have incorporated a mechanism of modulatory feedback allowing the reentry of signals from stages and representation higher up in the hierarchy that disambiguates the motion signal and spreads activities over longer distances. It is worth mentioning that their filtering mechanisms in V1 and MT could also be used in our model, but in order to focus on the influence of feedback processing, we omitted any additional parameters.

Nowlan and Sejnowski $[51,52]$ described a model of motion integration that utilized an explicit selection signal that is computed to determine the regions in the visual field where velocity estimations are most reliable. Motionsensitive cells are then gated by this signal to produce the final estimate. Note that the way they learn how to compute the selection signal is an elegant method that may be applied to learn a normalization process like the one described by Simoncelli and Heeger [50]. Our model differs in several ways from Nowlan's approach. While their approach utilizes a feedforward scheme, our model combines feedforward estimates with feedback integration and prediction. As a consequence, initial rough estimates are integrated and disambiguated over time within a recurrent loop of matching velocities and motion predictions generated in area MT. As a by-product, the determination of reliable motion estimates is computed implicitly in our model instead of explicitly generating a decision-like selection signal.

Several algorithms have been proposed in the framework of least-squares optimization [4] and regularization by incorporating a model smoothness (or prior) term to impose necessary constraints on the solution. The least-squares approach to motion estimation assumes locally constant motion patches to gather enough local motion measures over a small neighbourhood to disambiguate local motion estimates by an intersection-of-constraints (IOC) approach. Unlike the Lucas-Kanade (LK) approach the model architecture proposed here does not assume local constant motion 
over a predetermined neighbourhood. Instead, the continuous smoothing, subsequent amplification (through feedback signals), and the competitive interaction and normalization enhance arrangements of salient motion configurations in a stimulus adaptive fashion. Depending on the density of motion estimates the activity will be enhanced or lowered to keep the overall motion energy balanced. An IOC solution is computed when strong localized features will be detected in the signal and further enhanced and tracked over time. The stage of motion integration at the input stage of model area MT is similar to the approach of velocity summation or velocity averaging (VA). Together, the proposed network smoothly blends several properties of IOC, VA, and feature tracking to arrive at a proper motion response. Regularization approaches, on the other hand, utilize constant regularizers [2] or employ model dependent inhomogeneous smoothness priors [9] in order to yield coherent estimations of the input object motion. In many cases, such mechanisms tend to smooth the velocity field in an undesired fashion, since the smoothness constraints are controlled by localized mechanisms only. In our proposal, we demonstrate how context information can be delivered to selectively enhance (or gate) the bottom-up motion signals to incorporate intermediate interpretations of scenic motion patterns. Thus we claim that the proposed feedforward and feedback architecture provides a powerful processing framework for motion analysis and feature integration.

In a similar fashion (like IOC) Weiss and Fleet [5] and Weiss et al. [6] estimated the velocity of a moving object using a Bayesian approach. Here, the coherent motion of a moving shape is determined by maximizing the posterior probability of noisy, thus uncertain, velocity votes giving the detected image motions. Simoncelli [7] extended this approach to model different noise contributions in the estimation process. This formulation leads to a probability representation in velocity space for all measures of single moving objects. In the spirit of IOC computation, all probability distributions are multiplicatively combined, assuming independence of individual motion estimates, to arrive at the likelihood density of a priori motion estimates given the underlying image motions. In addition a giving prior of expected velocities in the scene can be employed by multiplying the likelihood density. The motion estimation is solved by maximizing the posterior from all individual measures. In our model, we do not directly combine all initial estimates, since this requires a priori knowledge about which moving parts in the stimulus belong together. Instead, we allow initial motion signals to be modulated by a predictive signal from the higher processing stage of area MT, which serves as a local prior that is adaptive over time. In order to achieve a global consistent estimate, this process is iterated to allow propagation of disambiguated motion signals along extended shape boundaries. Again, we also still do not assume that the motion is composed of local piecewise constant velocity of patches. Rather, the motion can vary smoothly in homogeneous regions whereas in the case of discontinuities enhances the motion signals along extended boundaries.
5.2.2. Models Using Feedback Mechanisms and Recurrent Interaction. Grossberg et al. [53] and Mingolla [54] presented a model of motion integration and segmentation in MT and MST based on inputs from the form pathway (modelled as the FACADE framework-Form-AndColor-And-DEpth) [55]. Berzhanskaya et al. [56] further extended this mechanism to distinguish between intrinsic and extrinsic terminator signals that occur in the case of several moving surfaces that mutually occlude each other. Such terminators resemble two-dimensional changes in the spatiotemporal profile of the intensity distribution. Grossberg and coworkers studied how motion signals from partly occluded patterns can be integrated and segregated in a recurrent fashion. In contrast to our approach, their feedback signals (from MST) inhibit MT activities and have a more global character due to the RF size of MST cells (depending on the stimulus, these RFs cover 50\% up to $100 \%$ of the entire stimulus). In their model MST cells compete in a winner-takes-all fashion such that their responses that signal the most prominent motion direction will be selected. The authors suggest that such a mechanism of feedback inhibition and selection also helps to solve the aperture problem using a decision-like mechanism through the inhibitory influence of global context information as delivered by large-spanning kernels [53, 54, 57]. Castet et al. [58] demonstrated that perceived speed varies as a function of line length and orientation which has been successfully simulated by Chey et al. [57] as an instantaneous property in the momentary speed representation. At a given orientation increasing the line length reduces the apparent speed as measured by the ratio between speed measures in direction orthogonal to line orientation versus actual speed in (horizontal) motion direction. Reference [59], on the other hand, investigated the deviations in pursuit eye movement performance when elongated bars of varying lengths were presented which should be tracked by eye. The direction selectivity of MT cells changes over time to compensate for the initial error in the pursuit signal. We predict that the resolution of uncertainty for the aperture problem in the representation at model MT should be largely independent of the length of the bar stimulus. We predict that this size invariance provides a means to handle shapes that appear at different images sizes_other things being equal. This property is due to the ability of establishing robust feature motion signals (e.g., at corners or line ends) which can help to disambiguate locally ambiguous signals in the spatial neighbourhood of localized features. Three functional components establish this functionality, namely, (i) by utilizing different spatial resolutions (in different model areas) to build a pyramid structure for integrating input activities from previous processing stages, (ii) through recurrent interaction of cells in different areas (MT and V1 in this case) having different receptive field sizes, and (iii) by propagating disambiguated feature responses (velocities) along extended boundaries to fill-in salient motion representations. This can be observed with the stimulus shown in Figure 6 such that the time for resolving the disambiguous motion for the moving rectangle varies with its image size. 
Lidén and Pack [60] proposed a model of recurrent lateral motion interactions, which is able to produce a traveling wave of motion activation to solve the aperture problem. Like the model framework outlined here they use the normalization similar to the mechanism described by Simoncelli and Heeger [50] to emphasize salient motion estimates. In contrast to our model scheme their normalization mechanism is not isotropic in velocity space. The propagation is done by recurrent lateral excitation leading to an unbounded filling-in process, which has to be constrained by long-range inhibition of motion cells of different directional selectivity and by a separately processed motion boundary signal. In the absence of concurrent motion signals from multiple objects, their model leads to completely filled-in motion fields, which must be gated by multiplying the input signal in order to display only relevant motion patterns. Conversely, our model implements a kind of "soft gating" by biasing the input signal during feedback processing and therefore produces spatially restricted motion estimates at all time steps without an explicit computation of motion or form boundaries.

Koechlin et al. [61] describe a model of motion integration along the V1-MT pathway that utilizes mechanisms of recurrent lateral interactions. Their model utilizes a multiplicative combination of feedforward input and the result of lateral integration. Salient motion features are emphasized through a stage of normalization, and the results of recurrent lateral modulation (gating) are used to propagate these features. Though these mechanisms seem to be rather similar compared to those proposed in our model, their realization and behaviour differ in many respects. For example, their gating process leads to strong inhibition of the input signal once the model has focused on one specific velocity while the stimulus changes to another velocity. Such lateral multiplication intensifies the winner-takes-all characteristic of their model [61] and makes it more vulnerable to outliers. Our model follows a gradual prediction-andcorrection philosophy realized by an excitatory modulation of feedforward input through feedback signals followed by a center-surround competitive mechanism to realize a biased competition. Essential to our model is the decoupling of processes into different areas with different RF sizes. This, in turn, provides an extended context sampled by the higher visual area and the ability to correct (bias) and disambiguate cell activities at earlier stages (that operate on higher spatial accuracy). Our proposed architecture is demonstrated to deal with large varieties of shape or object surface appearance providing a mechanism of size invariant motion integration.

5.3. Further Extensions of the Model Framework. The model framework outlined in this contribution has been developed over several years and has also been extended by the authors along several directions in order to improve the network functionality as well as the capability to explain experimental data. For example, one of the strengths of the modelling framework is the ability to incorporate other processingstreams into one common framework. For example, we have independently pursued several lines of investigation to combine form and motion streams for feature integration and segregation. In particular, Tlapale and coworkers have proposed a combined motion integration mechanism that is modulated by a gated diffusion mechanism based on luminance continuity [15]. In brief, a simplified channel for form processing has been employed here that utilizes bilateral filtering to adapt the spatial weighting functions depending on the continuity of the input luminance image. The concept of bilateral filtering has been proposed in the computer vision community for implementing a specific variant for adaptive and anisotropic diffusion filtering for denoising [62]. In the work of Tlapale et al. [15] the bilateral filtering mechanism is adopted to steer the integration of motion signals at the stage of model MT. Here, the kernel for motion integration $\left\{\Lambda_{\sigma}^{\mathrm{MT}} \stackrel{\mathbf{x}, \mathbf{v e l}}{*} y_{2}^{V 1}(\mathbf{x}, \mathbf{v}, t)\right\}$ that is employed for generating the input activity $y_{0}^{\mathrm{MT}}(\mathbf{x}, \mathbf{v}, t)$ after initial filtering at the stage of model MT is modulated by orientation selective form information. In order to achieve such a stimulus adaptive mechanism a steering kernel is incorporated to yield

$$
\begin{aligned}
\int_{\Omega} \Lambda_{\sigma}^{\mathrm{MT}}\left(\mathbf{x}-\mathbf{x}^{\prime}\right) \cdot \Psi\left(\theta-\angle\left(\mathbf{x}, \mathbf{x}^{\prime}\right)\right) \cdot \Lambda_{\sigma}^{V 2}\left(I(\mathbf{x})-I\left(\mathbf{x}^{\prime}\right)\right) \\
\cdot y_{2}^{V 1}\left(\mathbf{x}^{\prime}, \mathbf{v}, t\right) d \mathbf{x}^{\prime},
\end{aligned}
$$

where $\Psi\left(\theta-\angle\left(\mathbf{x}, \mathbf{x}^{\prime}\right)\right)$ penalizes deviations in orientation of the virtual line between target location and the position in the surround from the axis of an oriented integration kernel, while $\Lambda_{\sigma}^{V 2}\left(I(\mathbf{x})-I\left(\mathbf{x}^{\prime}\right)\right)$ measures photometric differences that are indicative of different surface patches.

Beck and Neumann [16] investigated a different route by using modulating input (at stage 2 of the cascade) that is generated by activity from long-range integration of boundaries in the form-sensitive pathway of areas V1 and V2 interactions. Similar to Berzhanskaya et al. [56] the model considers the functional role of the interaction between the motion and the form pathway of visual cortex. The extended model shows how the distributed representations of visual features motion, disparity, and form in areas V1, V2, and MT mutually interact to arrive at a coherent representation of moving surface patches. The issue of $2 \mathrm{D}$ extrinsic motion cues generated at occlusions is considered that have to be treated differently from 2D intrinsic motion features of the same object. The model by Beck and Neumann [16] suggests that junctions that are detected in the form pathway are necessary to generate a correct percept in the motion pathway.

In different attempt, the core architecture proposed in this paper has been extended to successfully deal with the problem of robust representation and segregation of transparent motion. Transparent and semitransparent motion occurs whenever multiple motions are presented in the same part of visual space moving in different directions or with different speeds. The model of Raudies and Neumann [17] investigates the necessary mechanisms underlying initial motion detection, the required representations for velocity coding, and the integration and segregation of motion stimuli to account for the perception of transparent motion. 


\section{Summary and Conclusion}

We presented a model of motion processing in areas V1 and MT capable of handling synthetic as well as artificial image sequences. The model incorporates several key properties, namely, initial detection of raw flow information, temporal spreading of reliable motion signals to gradually correct uncertain flow estimates, and the ability to sharply segregate regions of individual visual motion. The model architecture thus makes several new contributions to develop an architecture of general purpose motion processing that is inspired by the architecture and function of the visual system in primates. First, we propose a model of cortical feedforward and feedback processing in the dorsal pathway of motion integration implementing a neural hypothesis-test cycle of computation. Most importantly, the feedback mechanism is part of top-down modulatory enhancement of initial activities that match signal properties at a higher processing stage. Second, the disambiguation of initial estimates is solved by the interplay between top-down modulation and subsequent lateral competition. Consequently, the network dynamics propagate disambiguated motion signals along shape boundaries, thus realizing a guided filling-in process [63]. This mechanism is important in that it provides a means to process objects of different sizes in an invariant fashion. Third, the model serves as a link between physiological recordings (e.g., [41]) and psychophysical investigations of perceptual motion integration [42]. Beyond this, the model is able to process real-world stimulus sequences to yield accurate motion estimations. We believe that this further justifies the explanatory competence of key computational elements of the model, as most other biologically inspired models do not compare the quality of their results against other technical or nontechnical models.

In all, the proposed model provides further evidence for key computational principles that are involved in the cortical computation of sensory stimuli, their integration, and segregation. These key principles have been developed to explain mechanisms of form processing in boundary grouping and texture segregation $[24,25]$. Here, we now propose the same core mechanisms to account for the processing of temporally varying stimuli in the cortical motion pathway. Given the evidence gathered from our computational experiments, we claim that the early processing stages in visual cortex along the ventral and the parietal pathway are organized in a homologous fashion. Modulatory feedback and subsequent divisive inhibition realize a mechanism of biased competition already at an early stage with a similar behavior as the one proposed by [40] for attention mechanisms to filter out irrelevant information. We have proposed a concept of feedback as part of a layered structure and representation and presented an implementation of multiple loops of recurrent interaction whose dynamics realize multilevel cortical hypothesis testing cycles. The architecture has demonstrated its usefulness in that several improvements and model extensions have been proposed. As a consequence we suggest that the core mechanisms as presented in this paper can be used
TABLE 1: Parameter settings for the constants and kernel sizes used in the neural computational model.

\begin{tabular}{lc}
\hline$\kappa_{\mathrm{FB}}^{V 1}$ & 10 \\
$\alpha^{V 1 / \mathrm{MT}}$ & 0.015 \\
$\beta_{2}^{V 1 / \mathrm{MT}}$ & 1 \\
$\delta_{2}^{V 1 / \mathrm{MT}}$ & 20 \\
$\lambda_{2}^{V 1 / \mathrm{MT}}$ & 0.25 \\
$\mathrm{~V} 1: \mathrm{MT}$ & $5: 1$ \\
$\Lambda_{\sigma}^{V 1 \text {,surr }}$ & $\frac{1}{2 \pi 4^{2}} \exp \left(-\left(x^{2} / 2 \cdot 4^{2}\right)\right)$ \\
$\Lambda_{\sigma}^{\mathrm{MT}, \text { surr }}$ & 1 for $x=0$ \\
\hline
\end{tabular}

as basic building blocks that are already powerful to explain a wealth of empirical data and are also capable to process realistic sequences in technical applications. It is thus conceivable that other investigators interested in biologically inspired technology may start from this point in order to further develop mechanisms in this framework.

\section{Appendix}

Table 1 is included to display the parameters settings used for the computational simulations conducted in Section 5 .

The range of velocities tested for the correlation-based matching in the initial motion estimation have been limited to a range of $\pm(7,7)$ pix.

\section{Acknowledgments}

The authors would like to express their gratitude to the two anonymous reviewers for their thorough reading and constructive criticism on the first version of the manuscript. Their comments were very helpful to improve the manuscript. This joint research has been supported by the European Community in the 7th framework program ICT-project no. 215866-SEARISE. P.Kornprobst further acknowledges funding support by the Région Provence Alpes Côte d'Azur. H.Neumann and J.D.Bouecke are further supported by the Transregional Collaborative Research Center SFB/TRR 62 "Companion-Technology for Cognitive Technical Systems" funded by the German Research Foundation (DFG).

\section{References}

[1] H. R. Wilson, V. P. Ferrera, and C. Yo, "A psychophysically motivated model for two-dimensional motion perception," Visual Neuroscience, vol. 9, no. 1, pp. 79-97, 1992.

[2] B. K. P. Horn and B. G. Schunck, "Determining optical flow," Artificial Intelligence, vol. 17, no. 1-3, pp. 185-203, 1981.

[3] E. H. Adelson and J. A. Movshon, "Phenomenal coherence of moving visual patterns," Nature, vol. 300, no. 5892, pp. 523$525,1982$. 
[4] S. Baker and I. Matthews, "Lucas-Kanade 20 years on: a unifying framework, part 1," Carnegie-Mellon Univ., Robotics Institute, CMU-RI-TR-02-16, 2002, http://www.ri.cmu.edu/ cgi-bin/tech_reports.cgi.

[5] Y. Weiss and D. J. Fleet, "Velocity likelihoods in biological and machine vision," in Probabilistic Models of the Brain: Perception and Neural Function, R. P. N. Rao, B. A. Olshausen, and M. S. Lewicki, Eds., pp. 81-100, MIT Press, Cambridge, Mass, USA, 2001.

[6] Y. Weiss, E. P. Simoncelli, and E. H. Adelson, "Motion illusions as optimal percepts," Nature Neuroscience, vol. 5, no. 6, pp. 598-604, 2002.

[7] E. P. Simoncelli, "Bayesian multi-scale differential optical flow," in Handbook of Computer Vision and Applications, B. Jähne, H. Haussecker, and P. Geissler, Eds., vol. 2, chapter 14, pp. 297-422, Academic Press, New York, NY, USA, 1999.

[8] M. Del Viva and M. C. Morrone, "Motion analysis by feature tracking," Vision Research, vol. 38, no. 22, pp. 3633-3653, 1998.

[9] H. H. Nagel, "On the estimation of optical flow: relations between different approaches and some new results," Artificial Intelligence, vol. 33, no. 3, pp. 299-324, 1987.

[10] E. C. Hildreth, "Computations underlying the measurement of visual motion," Artificial Intelligence, vol. 23, no. 3, pp. 309354, 1984.

[11] D. C. Van Essen and J. L. Gallant, "Neural mechanisms of form and motion processing in the primate visual system," Neuron, vol. 13, no. 1, pp. 1-10, 1994.

[12] J. L. Barron, D. J. Fleet, and S. S. Beauchemin, "Performance of optical flow techniques," International Journal of Computer Vision, vol. 12, no. 1, pp. 43-77, 1994.

[13] S. Baker, S. Roth, D. Scharstein, M. J. Black, J. P. Lewis, and R. Szeliski, "A database and evaluation methodology for optical flow," in Proceedings of the 11th IEEE International Conference on Computer Vision (ICCV'07), October 2007.

[14] P. Bayerl and H. Neumann, "Disambiguating visual motion through contextual feedback modulation," Neural Computation, vol. 16, no. 10, pp. 2041-2066, 2004.

[15] É. Tlapale, G. S. Masson, and P. Kornprobst, "Modelling the dynamics of motion integration with a new luminance-gated diffusion mechanism," Vision Research, vol. 50, no. 17, pp. 1676-1692, 2010.

[16] C. Beck and H. Neumann, "Interactions of motion and form in visual cortex - a neural model," Journal of Physiology Paris, vol. 104, no. 1-2, pp. 61-70, 2010.

[17] F. Raudies and H. Neumann, "A model of neural mechanisms in monocular transparent motion perception," Journal of Physiology Paris, vol. 104, no. 1-2, pp. 71-83, 2010.

[18] M. Carandini, D. J. Heeger, and J. A. Movshon, "Linearity and normalization in simple cells of the macaque primary visual cortex," Journal of Neuroscience, vol. 17, no. 21, pp. 8621-8644, 1997.

[19] A. V. M. Herz, T. Gollisch, C. K. Machens, and D. Jaeger, "Modeling single-neuron dynamics and computations: a balance of detail and abstraction," Science, vol. 314, no. 5796, pp. 80-85, 2006.

[20] P. Dayan and L. F. Abbot, Theoretical Neuroscience, MIT Press, Cambridge, Mass, USA, 2001.

[21] S. J. Thorpe, "Localized versus distributed representations," in The Handbook of Brain Theory and Neural Networks, M. A. Arbib, Ed., MIT Press, Cambridge, Mass, USA, 2nd edition, 2003.
[22] A. K. Engel, P. R. Roelfsema, P. Fries, M. Brecht, and W. Singer, "Role of the temporal domain for response selection and perceptual binding," Cerebral Cortex, vol. 7, no. 6, pp. 571$582,1997$.

[23] S. Grossberg, "Nonlinear neural networks: principles, mechanisms, and architectures," Neural Networks, vol. 1, no. 1, pp. 17-61, 1988.

[24] H. Neumann and W. Sepp, "Recurrent V1-V2 interaction in early visual boundary processing," Biological Cybernetics, vol. 81, no. 5-6, pp. 425-444, 1999.

[25] A. Thielscher and H. Neumann, "Neural mechanisms of cortico-cortical interaction in texture boundary detection: a modeling approach," Neuroscience, vol. 122, no. 4, pp. 921939, 2003.

[26] A. Thielscher and H. Neumann, "A computational model to link psychophysics and cortical cell activation patterns in human texture processing," Journal of Computational Neuroscience, vol. 22, no. 3, pp. 255-282, 2007.

[27] P. R. Roelfsema, V. A. F. Lamme, H. Spekreijse, and H. Bosch, "Figure-ground segregation in a recurrent network architecture," Journal of Cognitive Neuroscience, vol. 14, no. 4, pp. 525-537, 2002.

[28] A. Angelucci, J. B. Levitt, E. J. S. Walton, J. M. Hupé, J. Bullier, and J. S. Lund, "Circuits for local and global signal integration in primary visual cortex," Journal of Neuroscience, vol. 22, no. 19, pp. 8633-8646, 2002.

[29] R. T. Born and D. C. Bradley, "Structure and function of visual area MT," Annual Review of Neuroscience, vol. 28, pp. 157-189, 2005.

[30] R. Eckhorn, H. J. Reitboeck, M. Arndt, and P. W. Dicke, "Feature linking via synchronization among distributed assemblies: simulations of results from cat visual cortex," Neural Computation, vol. 2, pp. 293-307, 1990.

[31] K. Zipser, V. A. F. Lamme, and P. H. Schiller, "Contextual modulation in primary visual cortes," Journal of Neuroscience, vol. 16, pp. 7376-7389, 1996.

[32] J. M. Hupé, A. C. James, P. Girard, S. G. Lomber, B. R. Payne, and J. Bullier, "Feedback connections act on the early part of the responses monkey visual cortex," Journal of Neurophysiology, vol. 85, no. 1, pp. 134-145, 2001.

[33] J. Bullier, "Hierarchies of Cortical Areas," in The Primate Visual System, J. H. Kaas and C. E. Collins, Eds., chapter 8, pp. 181-204, CRC Press, Boulder, Colo, USA, 2003.

[34] D. D. Stettler, A. Das, J. Bennett, and C. D. Gilbert, "Lateral connectivity and contextual interactions in macaque primary visual cortex," Neuron, vol. 36, no. 4, pp. 739-750, 2002.

[35] G. Sperling, "Model of visual adaptation and contrast detection," Perception \& Psychophysics, vol. 8, pp. 143-157, 1970.

[36] M. Carandini and D. J. Heeger, "Summation and division by neurons in primate visual cortex," Science, vol. 264, no. 5163, pp. 1333-1336, 1994.

[37] M. J. Escobar, G. S. Masson, T. Vieville, and P. Kornprobst, "Action recognition using a bio-inspired feedforward spiking network," International Journal of Computer Vision, vol. 82, no. 3, pp. 284-301, 2009.

[38] T. D. Albright and R. Desimone, "Local precision of visuotopic organization in the middle temporal area (MT) of the macaque," Experimental Brain Research, vol. 65, no. 3, pp. 582592, 1987.

[39] E. H. Adelson and J. R. Bergen, "Spatiotemporal energy models for the perception of motion," Journal of the Optical Society of America A, vol. 2, no. 2, pp. 284-299, 1985. 
[40] R. Desimone and J. Duncan, "Neural mechanisms of selective visual attention," Annual Review of Neuroscience, vol. 18, pp. 193-222, 1995.

[41] C. C. Pack and R. T. Born, “Temporal dynamics of a neural solution to the aperture problem in visual area MT of macaque brain," Nature, vol. 409, no. 6823, pp. 1040-1042, 2001.

[42] D. Williams and G. Phillips, "Cooperative phenomena in the perception of motion direction," Journal of the Optical Society of America A, vol. 4, no. 5, pp. 878-885, 1987.

[43] É. Tlapale, P. Kornprobst, J. D. Bouecke, H. Neumann, and G. S. Masson, "Towards a bio-inspired evaluation methodology for motion estimation models," INRIA Rapport de recherché 7317, June 2010.

[44] B. Lucas and T. Kanade, "An iterative image registration technique with an application to stereo vision," in Proceedings of the 7th International Joint Conference on Artificial Intelligence (IJCAI '81), pp. 674-679, Vancouver, BC, Canada, August 1981.

[45] J. H. R. Maunsell, “The brain's visual world: representation of visual targets in cerebral cortex," Science, vol. 270, no. 5237, pp. 764-769, 1995.

[46] J. H. R. Maunsell and D. C. Van Essen, "Functional properties of neurons in middle temporal visual area of the macaque monkey. I. Selectivity for stimulus direction, speed, and orientation," Journal of Neurophysiology, vol. 49, no. 5, pp. 1127-1147, 1983.

[47] J. A. Movshon, E. H. Adelson, M. S. Gizzi, and W. T. Newsome, "The analysis of moving visual patterns," in Pattern Recognition Mechanisms, C. Chagas, R. Gattass, and C. R. Gross, Eds., pp. 117-151, Vatican Press, Vatican City, 1985.

[48] C. C. Pack, M. S. Livingstone, K. R. Duffy, and R. T. Born, "End-stopping and the aperture problem: two-dimensional motion signals in macaque V1," Neuron, vol. 39, no. 4, pp. 671-680, 2003.

[49] K. J. Friston and C. Büchel, "Attentional modulation of effective connectivity from V2 to V5/MT in humans," Proceedings of the National Academy of Sciences of the United States of America, vol. 97, no. 13, pp. 7591-7596, 2000.

[50] E. P. Simoncelli and D. J. Heeger, "A model of neuronal responses in visual area MT," Vision Research, vol. 38, no. 5, pp. 743-761, 1998.

[51] S. J. Nowlan and T. J. Sejnowski, "Filter selection model for motion segmentation and velocity integration," Journal of the Optical Society of America A, vol. 11, no. 12, pp. 3177-3200, 1994.

[52] S. J. Nowlan and T. J. Sejnowski, "A selection model for motion processing in area MT of primates," Journal of Neuroscience, vol. 15, no. 2, pp. 1195-1214, 1995.

[53] S. Grossberg, E. Mingolla, and L. Viswanathan, "Neural dynamics of motion integration and segmentation within and across apertures," Vision Research, vol. 41, no. 19, pp. 2521$2553,2001$.

[54] E. Mingolla, "Neural models of motion integration and segmentation," Neural Networks, vol. 16, no. 5-6, pp. 939-945, 2003.

[55] S. Grossberg, "3-D vision and figure-ground separation by visual cortex," Perception and Psychophysics, vol. 55, no. 1, pp. 48-121, 1994.

[56] J. Berzhanskaya, S. Grossberg, and E. Mingolla, "Laminar cortical dynamics of visual form and motion interactions during coherent object motion perception," Spatial Vision, vol. 20, no. 4, pp. 337-395, 2007.
[57] J. Chey, S. Grossberg, and E. Mingolla, "Neural dynamics of motion grouping: from aperture ambiguity to object speed and direction," Journal of the Optical Society of America A, vol. 14, no. 10, pp. 2570-2594, 1997.

[58] E. Castet, J. Lorenceau, M. Shiffrar, and C. Bonnet, "Perceived speed of moving lines depends on orientation, length, speed and luminance," Vision Research, vol. 33, no. 14, pp. 19211936, 1993.

[59] R. T. Born, C. C. Pack, C. R. Ponce, and SI. Yi, “Temporal evolution of 2-dimensional direction signals used to guide eye movements," Journal of Neurophysiology, vol. 95, no. 1, pp. 284-300, 2006.

[60] L. Lidén and C. Pack, "The role of terminators and occlusion cues in motion integration and segmentation: a neural network model," Vision Research, vol. 39, no. 19, pp. 33013320, 1999.

[61] E. Koechlin, J. L. Anton, and Y. Burnod, "Bayesian inference in populations of cortical neurons: a model of motion integration and segmentation in area MT," Biological Cybernetics, vol. 80, no. 1, pp. 25-44, 1999.

[62] C. Tomasi and R. Manduchi, "Bilateral filtering for gray and color images," in Proceedings of the 6th IEEE International Conference on Computer Vision, pp. 839-846, Bombay, India, January 1998.

[63] H. Neumann, "Completion phenomena in vision: a computational approach," in Filling-in-From Perceptual Completion to Cortical Reorganization, L. Pessoa and P. De Weerd, Eds., pp. 151-173, Oxford University Press, New York, NY, USA, 2003. 